\title{
Exploiting symmetries in mathematical programming via orbital independence
}

\author{
Gustavo Dias · Leo Liberti
}

Received: date / Accepted: date

\begin{abstract}
The presence of symmetries in the solution set of mathematical programs requires the exploration of symmetric subtrees during the execution of Branch-andBound type algorithms and yields increases in computation times. When some of the solution symmetries are evident in the formulation, it is possible to deal with symmetries as a preprocessing step. In this sense, implementation-wise, one of the simplest approaches is to break symmetries by adjoining Symmetry-Breaking Constraints (SBCs) to the formulation. Designed to remove some of the symmetric global optima, these constraints are generated from each orbit of the action of the symmetries on the variable index set. Incompatible SBCs however make all of the global optima infeasible. In this paper we introduce and test a new concept of Orbital Independence which we define as independent sets of orbits. We provide necessary conditions for characterizing independent sets of orbits and also prove that such sets embed sufficient conditions to exploit symmetries from two or more distinct orbits concurrently. The theory developed is used to devise an algorithm that potentially identifies the largest independent set of orbits of any mathematical program. Extensive numerical experiments are provided to validate the theoretical results.
\end{abstract}

Keywords combinatorial optimization · symmetry breaking · group theory · quadratic programming

\section{Introduction}

Mathematical Programming (MP) is a descriptive language used to formalize several types of optimization problems by defining a class of corresponding mathematical

This paper is an extension of the work presented in [5].

Gustavo Dias

CNRS LIX, École Polytechnique, 91128 Palaiseau, France

E-mail: dias@lix.polytechnique.fr

Leo Liberti

CNRS LIX, École Polytechnique, 91128 Palaiseau, France

E-mail: liberti@lix.polytechnique.fr 
programs [15]. Let $\mathbb{M P}$ denote such a class. In this context, we consider problems $P \in \mathbb{M P}$ in the following general form:

$$
\left.\begin{array}{c}
\min _{x} f(x) \\
\forall i \in \mathcal{I}_{I} g_{i}(x) \leq 0, \\
\forall i \in \mathcal{I}_{E} g_{i}(x)=0, \\
x \in B
\end{array}\right\}
$$

In problem $P, f, g_{i}: \mathbb{R}^{n} \rightarrow \mathbb{R}$ are functions for which we have closed form expressions. The set $B$ might contain nonfunctional constraints such as ranges $\left[x^{L}, x^{U}\right]$ for the decision variables, and/or integrality constraints. For a mathematical program $P$ we let $\mathscr{F}(P)$ denote its feasible region and $\mathscr{G}(P)$ the set of its global optima.

Quite often researchers and practitioners face formulations $P$ which contain undesired mathematical properties. In such cases, casting the given problem $P$ into a different one, say $P^{\prime} \in \mathbb{M P}$, is a natural strategy: $P^{\prime}$ is called a reformulation of $P$. Any reformulation $P^{\prime}$ shares (numerical) properties with $P$ (e.g. the set of global optima), but is in some sense better than the original program. There are indeed many types of reformulations such as relaxations, approximations, variable changes, and all of them play an essential role in MP [15]. In our context, recall that the Branch-and-Bound (BB) algorithm paradigm is the most widely used technique for solving optimization problems formulated as $P$. Briefly, a tree-based search for global optima is performed in $\mathscr{F}(P)$. These algorithms may however converge slowly on problems whose feasible region has many symmetric global optima because all the symmetric leaf nodes in the BB tree must be visited in order to assert convergence. In fact, it was found that roughly $18 \%$ of the MP instances in commonly employed public libraries have nontrivial symmetry [16]. Symmetries are therefore investigated in MP mainly to reduce the computation time of BB type algorithms. In this sense, we aim to derive reformulations $P^{\prime}$ whose sets $\mathscr{G}\left(P^{\prime}\right)$ contain less symmetric global optima than $\mathscr{G}(P)$, meaning that $\mathscr{G}\left(P^{\prime}\right) \subseteq \mathscr{G}(P)$; and $P^{\prime}$ will be derived by addjoining Symmetry-Breaking Constraints (SBCs) to $P$.

In general, any strategy designed to cope with symmetries in MP can be divided into two main phases: (a) symmetry detection and (b) symmetry exploitation. When both phases (detection and exploitation) are performed before running the solution algorithm, we call such strategy a Static Symmetry Breaking (SSB) approach. Otherwise, a Dynamic Symmetry Breaking (DSB) approach [22]. This work concerns a general-purpose automated SSB strategy that advocates the usage of SBCs. Overall, we can further describe our methodology in a subtle higher level of detail w.r.t. the exploitation phase as follows: (a) detect formulation symmetries, (b) generate new constraints and (c) reformulate the original problem. The main contribution of this paper relates to the second step: constraint generation. Symmetry-breaking devices are usually derived from orbits of the action of the formulation group on the set of decision variables; however, one cannot simply use such devices for all the orbits simultaneously because some orbits depend on each other in a very precise mathematical sense. We devise herein a concept of Orbital Independence (OI) called independent set of orbits which allow us to overcome this limitation. We provide necessary conditions for characterizing independent sets of orbits and also prove that such sets embed sufficient conditions to exploit symmetries from two or more distinct orbits concurrently.

More precisely, this paper extends the content of [5] in three fronts: first we characterize independent sets of orbits via direct product of groups (Lemma 3) and prove 
that the OI conditions provided by Corollary 1 (see Section 3.3) are not sufficient to characterize independent sets of orbits (Example 2); second, we introduce a family of highly symmetric Binary Quadratic Programs (denoted by $\mathbb{B Q P})$; and lastly, besides the original tests using symmetric instances from MIPLIB2010, we enlarge our dataset with symmetric instances from MINLPLib2 and randomly generated symmetric Binary Quadratic Programs.

The rest of the paper is organized as follows: in Section 2 we introduce notation, recall concepts of Group Theory and review some previous work related to symmetries in MP; in Section 3 we present all the theoretical developments concerning the OI framework; in Section 4 we describe in details the SBCs generation algorithm devised based on the recently constructed theory; and finally, computational experiments are provided and analysed in Section 5.

\section{Notation and previous work}

\subsection{Group Theory}

We consider that permutation groups act on vectors in $\mathbb{R}^{n}$ by permuting its components and that permutations act on sets of vectors by acting on each vector individually. For a vector $v \in \mathbb{R}^{n}$ and a subset $N \subseteq[n]=[1, \ldots, n]$, we let $v[N]$ denote the projection of $v$ on the coordinates indexed by $N$.

Nomenclature-wise, $S_{n}$ and $C_{n}$ are the symmetric and cyclic group of order $n$, respectively. $\operatorname{Sym}(X)$ is the symmetric group on a set $X$ (e.g. $\left.S_{n}=\operatorname{Sym}([n])\right)$. Throughout the paper, let $H$ and $G$ denote permutation groups and $\diamond$ denote the group operator. If $H$ is a subgroup of $G$, we write $H \leq G$. If $H$ is isomorphic to $G$, we write $H \cong G$. If $H$ is a normal subgroup of $G$, we write $H \triangleleft G$. $\langle\Delta\rangle$ denotes the group generated by the set $\Delta$ of generators and $G \times H$ denotes the direct product of groups.

Consider a set $X$. We recall that an orbit is an equivalence class of the quotient set $X / \sim$, where $i \sim j$ if there is a permutation $g \in G$ such that $g(i)=j$. This way, the group $G$ partitions $X$ into a set $\Omega_{G}$ of orbits $\omega_{1}, \ldots, \omega_{p}$, for $p \in\left[1, \ldots,\left|\Omega_{G}\right|\right]$. Moreover, we let $\omega(\ell)$ denote the $\ell$-th element of $\omega$ (stored as a list).

Let $Y \subseteq X$. The pointwise stabilizer of $Y$ w.r.t. $G$ is the subgroup of permutations of $G$ fixing each element of $Y$, i.e., $G^{Y}=\{g \in G \mid \forall y \in Y(g \diamond y=y)\}$. The setwise stabilizer of $Y$ w.r.t. $G$ is the subgroup of those permutations of $G$ under which $Y$ is invariant, i.e., $\operatorname{stab}(Y, G)=\{g \in G \mid \forall y \in Y(g \diamond y \in Y)\}$.

A group action is transitive on a set $X$ if $s \sim t$ for each $s, t \in X$. If a group $G$ acts transitively on a set $X$, then $X$ is an orbit of $G$.

\subsection{Literature review}

Apart from problem oriented breaking techniques [17], most of the work regarding symmetries in MP was dedicated to develop general-purpose symmetry group computations and breaking techniques embedded in BB-type algorithms. The work in this sense follows three main streams.

The first is devoted to DSB techniques and was established by Margot [20,21]. He defined the relaxation group of Binary Linear Programs (denoted BLP) and used 
it to derive pruning strategies and cuts by means of isomorphism; this technique is known as isomorphism prunning. The idea was later extended and named orbital branching in [25] by using valid disjunctions to orbits of the formulation group to derive $\mathrm{BB}$ braching rules.

The second refers to SSB techniques and the usage of SBCs to tighten the search space. It was established by Kaibel and coworkers $[14,6]$, with the introduction of the packing and partitioning orbitopes. Inspired by orbitopes, Friedman proposed a more general approach named fundamental domains [10]. Liberti then studied and extended the use of general purpose SBCs to Mixed-Integer Nonlinear Programs (denoted by $\mathbb{M I N L} \mathbb{P})$ in $[17,16]$.

Developed at first to Mixed-Integer Linear Programs (denoted by $\mathbb{M I L I}$ ), the third stream (named orbital shrinking by Fischetti and Liberti [7]) focus on deriving compact symmetry free relaxations by replacing whole orbits by single variables. The technique was extended to convex $\mathbb{M I N L} \mathbb{P}_{s}$ and some nonconvex $\mathbb{M I N L} \mathbb{P}_{s}$ having a special structure. A recent survey on the subject is available in [8].

As concerns symmetry detection, the formal description of $P$ in the language $\mathcal{L}$ can be parsed into a Directed Acyclic Graph (DAG) data structure $T$ using a fairly simple context-free grammar [2]; we refer to [4] for further details and an example about this procedure. In practice, one can write $P$ using a modelling language such as AMPL [9] and use an unpublished AMPL API to derive $T$ and its set of nodes $V(T)$ [11]. Since $T$ is a labelled graph, we know how to compute the group $\mathcal{G}$ of its label-invariant isomorphisms [23,24]. Furthermore, it was shown in [16] that the action of $\mathcal{G}$ can be projected to the leaf nodes of $V(T)$, which represent the set of decision variables of $P$, and that this projection induces a group homomorphism $\phi$ mapping $\mathcal{G}$ to a certain group image known as the formulation group of $P$.

Definition 1 The formulation group $G_{P}$ of $P$ is the group of permutations which acts on the set of decision variables of $P$ while keeping the objective function $f(x)$ and the feasible region $\left\{g_{i}(x) \mid i \in \mathcal{I}_{I} \cup \mathcal{I}_{E}\right\}$ unchanged.

The solution group of $P$ is the group of permutations which keeps the set $\mathscr{G}(P)$ invariant, i.e. $G_{P}^{*}=\operatorname{stab}\left(\mathscr{G}_{P}, S_{n}\right)$. It is easy to show that $G_{P} \leq G_{P}^{*}$. It is impractical however to compute solution groups because it requires aprioristic knowledge of $\mathscr{G}_{P}$. On the other hand, if $G_{P}$ is nontrivial, one can use the methodology proposed in [16] to computing generators for $G_{P}$ and extract symmetries from $P$ prior to solving it. In $[16, \S 8.3]$ one can find many examples of formulation groups that operate in MP, such as symmetric, cyclic, dihedral and groups which are represented by means of the direct product operator. Moreover, it is important to note that formulation groups act on the set of decision variables only because mathematical programs are invariant under constraint-order permutations.

Symmetry is exploited in MP in a number of different ways, however their most efficient exploitation appears to be their usage within DSB strategies [20,21,25]. Such approaches are, unfortunately, difficult to implement, as each solver code must be addressed separately. Their simplest exploitation is the SSB approach $[22, \S 8.2]$ which consists in adjoining some SBCs to the original formulation $P$ in the hope of making all but one of the symmetric global optima infeasible. This procedure yields a reformulation of the narrowing type [15].

Definition 2 Given a problem $P$, a narrowing $P^{\prime}$ of $P$ is such that (a) there is a function $\eta: \mathscr{F}\left(P^{\prime}\right) \rightarrow \mathscr{F}(P)$ for which $\eta\left(\mathscr{G}\left(P^{\prime}\right)\right) \subseteq \eta(\mathscr{G}(P))$ and $(\mathrm{b}) P^{\prime}$ is infeasible only if $P$ is. 
Following the usual trade-off between efficiency and generality, approaches which offer provable guarantees of removing symmetric optima are limited to special structures [14], whereas approaches which hold for any mathematical program in the large class $P$ are mostly common-sense constraints designed to work in general [17].

As concerns symmetry exploitation via SBCs, the projection homomorphism $\phi$ defined above for $\mathcal{G}$ and the leaf nodes of the parsing tree can be restricted to act on $G_{P}$ and generalized to project its action to any subset $Y \subseteq X$. Let $\phi_{Y}$ denote this generalized action projection homomorphism, which is defined as follows: for each $\pi \in G_{P}, \phi_{Y}(\pi)$ is the product of the cycles of $\pi$ having all components in $Y$. If $Y$ is some orbit $\omega \in \Omega_{G_{P}}$, then (a) the image of $\phi_{Y}$ is a group $G_{P}[\omega]$ called the transitive constituent of $\omega$, (b) $G^{Y}$ is the kernel of $\phi_{Y}$ and (c) $\operatorname{stab}(Y, G)=G$.

Now let $x^{*} \in \mathscr{G}(P)$. If $G_{P}[\omega] \cong \operatorname{Sym}(\omega)$ on the orbit, $\mathscr{G}(P)$ contains vectors which yield every possible order of $x^{*}[\omega]$ when projected onto $\omega$. Thus we can arbitrarily choose one order, e.g.:

$$
\forall \ell<|\omega| \quad x_{\omega(\ell)} \leq x_{\omega(\ell+1)}
$$

enforce this order by means of SBCs, and still be sure that at least one global optimum remains feasible. The constraints in Eq. (1) are called strong $S B C s$. If $G_{P}[\omega]$ has any other group structure, we observe that, by transitivity of the transitive constituent, at least one permutation in $G_{P}[\omega]$ will map the component having minimum value in $x^{*}[\omega]$ to the first component. This yields the weak SBCs:

$$
\forall \ell \in \omega \backslash\{\omega(1)\} \quad x_{\omega(1)} \leq x_{\omega(\ell)} .
$$

Strong SBCs select one order out of $|\omega|$ ! many, and hence are able to break all the symmetries in $G_{P}[\omega]$. Weak SBCs may unlikely achieve that. We let $g(x[N]) \leq 0$ denote SBCs involving only variables $x_{j}$ with $j$ in the set $N$.

Remark 1 The choice of minimum value and first components are arbitrary; alternative sets of SBCs can occur for other distinct choices.

Lastly, we refer readers to surveys $[22,17]$ for an assessment of the state of the art in symmetry handling methods in Mathematical Programming.

\section{Orbital independence}

In this section we start introducing our OI theoretical results. First we exemplify how incompatible SBCs cut global optima from a mathematical program; then we recall the OI conditions originally introduced in [16] and [19]; finally we introduce the concept of independent set of orbits and the conditions which we shall use to identify such sets within the algorithmic framework presented in Section 4 .

\subsection{Incompatible SBCs}

In general, one may only adjoin to $P$ the SBCs associated to one single orbit. Example 1 shows that adjoining SBCs from two or more orbits chosen arbitrarily may result in all global optima being infeasible. 
Example 1 Let $P$ be the following $\mathbb{M L L P}$ :

$$
\begin{aligned}
\min _{x \in\{0,1\}^{4}} x_{1}+x_{2}+2 x_{3}+2 x_{4} \\
\quad\left(\begin{array}{cccc}
1 & 1 & 0 & 0 \\
0 & 0 & 1 & 1 \\
-1 & 0 & -1 & 0 \\
0 & -1 & 0 & -1
\end{array}\right)\left(\begin{array}{l}
x_{1} \\
x_{2} \\
x_{3} \\
x_{4}
\end{array}\right) \leq\left(\begin{array}{c}
1 \\
1 \\
-1 \\
-1
\end{array}\right) .
\end{aligned}
$$

This problem has as set of optima $\mathscr{G}(P)=\{(0,1,1,0),(1,0,0,1)\}$. In addition, it has formulation group $G_{P}=\langle(12)(34)\rangle$ and orbits $\Omega_{G_{P}}=\left\{\omega_{1}, \omega_{2}\right\}=\{\{1,2\},\{3,4\}\}$. Valid SBCs for $\omega_{1}$ (resp. $\omega_{2}$ ) are $x_{1} \leq x_{2}$ (resp. $x_{3} \leq x_{4}$ ). By simple inspection of the optima set, whereas adjoining either of the two SBCs yields valid narrowings, adjoining both simultaneously leads to an infeasible mathematical program.

\subsection{Some existing OI conditions}

Sufficient conditions to concurrently combine sets of SBCs generated by two different orbits, say $\omega, \theta \in \Omega_{G_{P}}$, into a valid narrowing of $\mathbb{M I N L} \mathbb{P}$ s are provided in [16]: (a) there is a subgroup $H \leq G_{P}[\omega \cup \theta]$ such that $H[\omega] \cong C_{|\omega|}$ and $H[\theta] \cong C_{|\theta|}$ and (b) $\operatorname{gcd}(|\omega|,|\theta|)=1$. Two orbits with these properties are called coprime. The author proposes an algorithm that interatively builds a set $\Omega_{C}$ of pairwise coprime orbits: at iteration $k$, an orbit $\omega^{k}$ is randomly picked and tested against coprime orbits found in previous iterations; should $\omega^{k}$ pairwisely satisfy the conditions above w.r.t. all previously found coprime orbits, it is added to $\Omega_{C}$. Once $\Omega_{C}$ is built, SBCs are generated for all of the orbits within it. The coprime conditions however are very restrictive and occur relatively rarely in practice, meaning that in general, most of information regarding the orbits remains unexploited.

A strategy based on chains of stabilizers is derived to overcome the limitations of the coprime narrowing in [19]. Based on the result that the map $\cdot[\omega]: G \rightarrow G[\omega]$ is a group homomorphism whose kernel is the pointwise stabilizer $G^{\omega}$, which is therefore a normal subgroup of $G$, the authors propose an algorithm that, at each iteration $k$, identifies the set of orbits resulting from the action of a group $G_{k}$ and randomly picks one of them to generate SBCs. The idea underlying this approach is how $G_{k}$ is updated in between iterations: if $\omega^{k}$ is the orbit used to generate SBCs at iteration $k, G_{k+1}=G^{\omega^{k}} \triangleleft G_{k}$. Provided that $G_{k+1}$ stabilizes $\omega^{k}$ pointwise and is a normal subgroup of $G_{k}, \omega^{k}$ is permanently factored out during the remaining iterations. The algorithm iterates until $G_{k}$ becomes trivial, meaning that the stabilizers'chain was totally exploited.

Remark 2 In both methods, the orbits are arbitrarily chosen at each iteration; for the same mathematical program, different orbit choices lead to different sets of SBCs.

\subsection{New conditions for OI}

We will now build the concept of independent set of orbits and provide necessary conditions for characterizing such sets. 
First, let $\omega, \theta \in \Omega_{G_{P}}$. We look at what happens to $\omega$ when $\theta$ is pointwise stabilized: either $G^{\theta}$ fixes $\omega$, or a subset of $\omega$, or it does not fix any element of $\omega$ at all. We can thus state the following binary dependence relations on the set $\Omega_{G_{P}}$.

Definition 3 The orbit $\omega$ is:

(a) dependent of $\theta$ (denoted by $\omega \rightarrow \theta$ ) if, for any subset $\sigma \subseteq \omega, \sigma \notin \Omega_{G^{\theta}}$;

(b) semi-dependent of $\theta$ (denoted by $\omega \rightsquigarrow \theta$ ) if there is at least one subset $\sigma \subsetneq \omega$ such that $\sigma \in \Omega_{G^{\theta}}$;

(c) independent of $\theta$ (denoted by $\omega\left\llcorner\theta\right.$ ) if $\omega \in \Omega_{G^{\theta}}$.

Next, let $\Gamma^{\omega}$ be the set of permutations of $G_{P}$ which move elements of the orbit $\omega$ nontrivially. By definition, $\Gamma^{\omega}$ does not contain the identity permutation $e$ of $G_{P}$ and thus it is not itself a group. Moreover, the following properties trivially hold: (a) $G^{\omega} \cap \Gamma^{\omega}=\varnothing$, (b) $\operatorname{stab}\left(\omega, G_{P}\right)=G^{\omega} \cup \Gamma^{\omega}=G_{P}$ and (c), for $\omega \in \Omega_{G_{P}}$, $G_{P}[\omega]=\phi_{\omega}\left(\Gamma^{\omega}\right) \cup\{e\}$.

Theorem 1 establishes the dependence relation between two orbits $\omega, \theta \in \Omega_{G_{P}}$ by comparing the sets $\Gamma^{\omega}$ and $\Gamma^{\theta}$.

Theorem 1 The following statements are true:

(1) If $\Gamma^{\theta}=\Gamma^{\omega}$ then $\theta \rightarrow \omega$ and $\omega \rightarrow \theta$;

(2) If $\Gamma^{\theta} \subset \Gamma^{\omega}$ then $\theta \rightarrow \omega$ and either $\omega\llcorner\theta$ or $\omega \rightsquigarrow \theta$;

(3) If $\Gamma^{\theta} \cap \Gamma^{\omega} \neq \varnothing$ then $(\theta\llcorner\omega$ or $\theta \rightsquigarrow \omega)$ and $(\omega\llcorner\theta$ or $\omega \rightsquigarrow \theta)$;

(4) If $\Gamma^{\theta} \cap \Gamma^{\omega}=\varnothing$ then $\theta\llcorner\omega$ and $\omega\llcorner\theta$.

Proof (1) Assume $\Gamma^{\theta}=\Gamma^{\omega}$ and consider $\omega$. Then $G^{\omega}=G_{P} \backslash \Gamma^{\omega} \Rightarrow G^{\omega} \cap \Gamma^{\theta}=$ $\varnothing \Rightarrow \theta \notin \Omega_{G^{\omega}}$ and $\theta \rightarrow \omega$. Since the same argument holds if we consider $\theta$, we also have $\omega \rightarrow \theta$.

(2) Assume $\Gamma^{\theta} \subset \Gamma^{\omega}$ and consider $\omega$. Then $G^{\omega}=G_{P} \backslash \Gamma^{\omega} \Rightarrow G^{\omega} \cap \Gamma^{\theta}=\varnothing \Rightarrow$ $\theta \notin \Omega_{G^{\omega}}$ and $\theta \rightarrow \omega$. Considering $\theta$, we have that $G^{\theta}=G_{P} \backslash \Gamma^{\theta} \Rightarrow G^{\theta} \cap \Gamma^{\omega} \neq \varnothing$. If the action of $G^{\theta}$ is transitive on $\omega$, we have $\omega\llcorner\theta$. Otherwise, we have $\omega \rightsquigarrow \theta$.

(3) Assume $\Gamma^{\theta} \cap \Gamma^{\omega} \neq \varnothing$ but neither set is wholly contained in the other, and consider $\omega$. Then $G^{\omega}=G_{P} \backslash \Gamma^{\omega} \Rightarrow G^{\omega} \cap \Gamma^{\theta} \neq \varnothing$. If the action of $G^{\omega}$ is transitive on $\theta$, we have $\theta\llcorner\omega$. Otherwise, we have $\theta \rightsquigarrow \omega$. The same argument holds if we consider $\theta$.

(4) Assume $\Gamma^{\theta} \cap \Gamma^{\omega}=\varnothing$ and consider $\omega$. Then $G^{\omega}=G_{P} \backslash \Gamma^{\omega} \Rightarrow G^{\omega} \supset \Gamma^{\theta} \Rightarrow$ $\theta \in \Omega_{G \omega}$ and $\theta\llcorner\omega$. The same holds if we consider $\theta$, thus $\omega\llcorner\theta$.

Lemma 1 The premise $\Gamma^{\theta} \cap \Gamma^{\omega}=\varnothing$ to condition (4) in Theorem 1 never holds.

Proof Let $\Delta$ be a set of generators of $G_{P}$. If there is a permutation $g \in \Delta$ such that $g[\omega]$ and $g[\theta]$ are nontrivial, then $g \in \Gamma^{\theta} \cap \Gamma^{\omega}$. Otherwise, let $\Delta^{\theta}=\{g \in \Delta \mid g[\omega]=e\}$ and $\Delta^{\omega}=\{g \in \Delta \mid g[\theta]=e\}$. Because every element of $G_{P}$ can be expressed as the combination (under the group operation) of finitely many elements of $\Delta$, there is $g \in G_{P}$ such that $g=g_{\omega} \diamond g_{\theta}$ where $g_{\omega} \in \Delta^{\omega}$ and $g_{\theta} \in \Delta^{\theta}$. Thus $g \in \Gamma^{\theta} \cap \Gamma^{\omega}$.

Based on the above definitions and results, the following lemma holds.

Lemma 2 The following statements are true:

(1) The relation $\rightarrow$ is reflexive and the relations $\rightsquigarrow$ and $\rightarrow$ are irreflexive;

(2) The relation $\rightarrow$ is symmetric iff $\Gamma^{\theta}=\Gamma^{\omega}$ and asymmetric iff $\Gamma^{\theta} \subset \Gamma^{\omega}$; 
(3) The relation $\rightarrow$ is transitive.

Proof The proof of statements (1) and (2) follows directly from Theorem 1. As concerns (3), let $\theta, \omega, \tau \in \Omega_{G_{P}}$ be distinct orbits satisfying $\theta \rightarrow \omega$ and $\omega \rightarrow \tau$. From Theorem $1, \theta \rightarrow \omega$ implies that either $\Gamma^{\theta}=\Gamma^{\omega}$ or $\Gamma^{\theta} \subset \Gamma^{\omega}$. Similarly, $\omega \rightarrow \tau$ implies that either $\Gamma^{\omega}=\Gamma^{\tau}$ or $\Gamma^{\omega} \subset \Gamma^{\tau}$. Then:

(a) $\Gamma^{\theta}=\Gamma^{\omega} \wedge \Gamma^{\omega}=\Gamma^{\tau} \Rightarrow \Gamma^{\theta}=\Gamma^{\tau} \Rightarrow \theta \rightarrow \tau$;

(b) $\Gamma^{\theta}=\Gamma^{\omega} \wedge \Gamma^{\omega} \subset \Gamma^{\tau} \Rightarrow \Gamma^{\theta} \subset \Gamma^{\tau} \Rightarrow \theta \rightarrow \tau$;

(c) $\Gamma^{\theta} \subset \Gamma^{\omega} \wedge \Gamma^{\omega}=\Gamma^{\tau} \Rightarrow \Gamma^{\theta} \subset \Gamma^{\tau} \Rightarrow \theta \rightarrow \tau$;

(d) $\Gamma^{\theta} \subset \Gamma^{\omega} \wedge \Gamma^{\omega} \subset \Gamma^{\tau} \Rightarrow \Gamma^{\theta} \subset \Gamma^{\tau} \Rightarrow \theta \rightarrow \tau$.

Whenever the dependence relations are symmetric, we write $\omega \leftrightarrow \theta$ or $\omega \leadsto \theta$ or $\omega$. Using this notation, we set forth that:

Definition 4 Two orbits $\omega, \theta \in \Omega_{G_{P}}$ are dependent if $\omega \leftrightarrow \theta$, semi-dependent if $\omega \rightsquigarrow \theta$ and independent if $\omega \leftrightarrow t \rightarrow \theta$.

Now we describe the independence relation ( $)$ ) by means of the direct product of groups. Let $H_{\omega}$ denote a group that acts transitively on $\omega$ and $G \diamond H$ the set of permutations generated by multiplying each permutation (except the identity) of $G$ by each permutation (except the identity) of $H$.

Lemma 3 For $\omega, \theta \in \Omega_{G_{P}}$, if there is a subgroup $H \leq G_{P}[\omega \cup \theta]$ such that $H=$ $H_{\omega} \times H_{\theta}$, then $\omega$ ل ل

Proof Assume that there is such a group $H$. Applying the definition of direct product of groups, we obtain that

$$
H_{\omega} \cup H_{\theta} \cup H_{\omega} \diamond H_{\theta} \leq G_{P}[\omega \cup \theta] .
$$

Moreover, we know that $G_{P}[\omega \cup \theta]=\phi_{\omega \cup \theta}\left(\Gamma^{\omega} \cup \Gamma^{\theta}\right) \cup\{e\}$. Using elementary set theory, we can write that $\Gamma^{\theta} \cup \Gamma^{\omega}=\left(G^{\theta} \cap \Gamma^{\omega}\right) \cup\left(G^{\omega} \cap \Gamma^{\theta}\right) \cup\left(\Gamma^{\omega} \cap \Gamma^{\theta}\right)$. Thus $\phi_{\omega \cup \theta}\left(\Gamma^{\omega} \cup \Gamma^{\theta}\right)=\phi_{\omega \cup \theta}\left(G^{\theta} \cap \Gamma^{\omega}\right) \cup \phi_{\omega \cup \theta}\left(G^{\omega} \cap \Gamma^{\theta}\right) \cup \phi_{\omega \cup \theta}\left(\Gamma^{\omega} \cap \Gamma^{\theta}\right)=\phi_{\omega}\left(G^{\theta} \cap \Gamma^{\omega}\right) \cup$ $\phi_{\theta}\left(G^{\omega} \cap \Gamma^{\theta}\right) \cup \phi_{\omega \cup \theta}\left(\Gamma^{\omega} \cap \Gamma^{\theta}\right)$ since $G^{\theta} \cap \Gamma^{\omega}$ stabilizes $\theta$ and $G^{\omega} \cap \Gamma^{\theta}$ stabilizes $\omega$; we then have that

$$
G_{P}[\omega \cup \theta]=\phi_{\omega}\left(G^{\theta} \cap \Gamma^{\omega}\right) \cup \phi_{\theta}\left(G^{\omega} \cap \Gamma^{\theta}\right) \cup \phi_{\omega \cup \theta}\left(\Gamma^{\omega} \cap \Gamma^{\theta}\right) \cup\{e\} .
$$

Comparing both expressions involving $G_{P}[\omega \cup \theta]$, we get by inclusion that $H_{\omega} \leq$ $\phi_{\omega}\left(G^{\theta} \cap \Gamma^{\omega}\right) \cup\{e\}, H_{\theta} \leq \phi_{\theta}\left(G^{\omega} \cap \Gamma^{\theta}\right) \cup\{e\}$ and $H_{\omega} \diamond H_{\theta} \leq \phi_{\omega \cup \theta}\left(\Gamma^{\omega} \cap \Gamma^{\theta}\right)$. Thus $G^{\theta} \cap \Gamma^{\omega} \neq \varnothing, G^{\omega} \cap \Gamma^{\theta} \neq \varnothing$ and $\Gamma^{\omega} \cap \Gamma^{\theta} \neq \varnothing$ since $H_{\omega}$ and $H_{\theta}$ are nontrivial groups by assumption, meaning that premise (3) in Theorem 1 holds. Moreover, $G^{\theta} \cap \Gamma^{\omega}$ acts transitively on $\omega$ and $G^{\omega} \cap \Gamma^{\theta}$ on $\theta$, which means that the action of the stabilizers $G^{\theta}$ and $G^{\omega}$ is also transitive on $\omega$ and $\theta$, respectively, since $G^{\theta} \cap \Gamma^{\omega} \leq G^{\theta}$ and $G^{\omega} \cap \Gamma^{\theta} \leq G^{\omega}$. Thus $\omega$ ل ا

Note the similarity between the conditions presented in Lemma 3 and the coprime conditions (Section 3.2), the latter being more restrictive. However, from a computational point of view, to the best of our knowledge, there is no method available in the literature capable of efficiently finding a subgroup $H \leq G[\omega \cup \theta]$ satisfying Lemma 3 for given orbits $\omega, \theta \in \Omega_{G_{P}}$. This is why we resort to characterize the OI conditions via pointwise stabilizers. 
Following, we extend the dependence relations presented above to sets of orbits. In this sense, consider a set $\Omega \subseteq \Omega_{G_{P}}$ and let $\Omega^{\omega}=\Omega \backslash \omega$ for $\omega \in \Omega$. The pointwise stabilizer of a set $\Omega$ of orbits is denoted as $G^{\Omega}$ hereafter. We look at what happens to $\omega$ when the set $\Omega^{\omega}$ is pointwise stabilized (i.e. when all the orbits in $\Omega^{\omega}$ are simultaneously pointwise stabilized) and, as previously, state suitable dependence definitions.

Definition 5 The orbit $\omega$ is:

(a) dependent of $\Omega^{\omega}$ (denoted by $\omega \hookrightarrow \Omega^{\omega}$ ) if, for any subset $\sigma \subseteq \omega, \sigma \notin \Omega_{G^{\Omega^{\omega}}}$;

(b) semi-dependent of $\Omega^{\omega}$ (denoted by $\omega \sim \Omega^{\omega}$ ) if there is at least one subset $\sigma \subsetneq \omega$ such that $\sigma \in \Omega_{G^{\Omega^{\omega}}}$;

(c) independent of $\Omega^{\omega}$ (denoted by $\omega \nrightarrow \Omega^{\omega}$ ) if $\omega \in \Omega_{G^{\Omega^{\omega}}}$.

Lemma 4 establishes necessary conditions to have $\omega \nrightarrow \Omega^{\omega}$.

Lemma 4 If $\omega \nrightarrow \Omega^{\omega}$, then $\omega\left\llcorner\theta\right.$ for all $\theta \in \Omega^{\omega}$.

Proof By definition, $\omega \nrightarrow \Omega^{\omega}$ implies that the action of $G^{\Omega^{\omega}}$ on $\omega$ is transitive. Since $G^{\Omega^{\omega}}$ is a subgroup of $G^{\theta}$ for every $\theta \in \Omega^{\omega}, G^{\theta}$ also acts transitively on $\omega$ and thus $\omega \hookrightarrow \theta$.

Now we can define an independent set of orbits. Note that similar definitions can be laid down as for dependent and semi-dependent sets of orbits.

Definition 6 A set $\Omega$ of orbits is said to be independent if $\omega \nrightarrow \Omega^{\omega}$ for all $\omega \in \Omega$.

Corollary 1 provides necessary conditions so as to a set of orbits be independent.

Corollary 1 If the set $\Omega$ of orbits is independent, then $\omega$ for all $\omega, \theta \in \Omega$.

Proof By Definition 6 and Lemma 4.

And Example 2 proves that the conditions in Corollary 1 are not sufficient to guarantee that a set $\Omega$ of orbits is independent.

Example 2 Let $P$ be the following $\mathbb{M I L I P}$ :

$$
\begin{aligned}
\min _{x \in\{0,1\}^{6}} & x_{1}+x_{2}+2 x_{3}+2 x_{4}+3 x_{5}+3 x_{6} \\
& \left(\begin{array}{llllll}
1 & 1 & 0 & 0 & 0 & 0 \\
0 & 0 & 1 & 1 & 0 & 0 \\
0 & 0 & 0 & 0 & 1 & 1 \\
1 & 0 & 1 & 0 & 1 & 0 \\
0 & 1 & 0 & 1 & 1 & 0 \\
1 & 0 & 0 & 1 & 0 & 1 \\
0 & 1 & 1 & 0 & 0 & 1
\end{array}\right)\left(\begin{array}{l}
x_{1} \\
x_{2} \\
x_{3} \\
x_{4} \\
x_{5} \\
x_{6}
\end{array}\right) \leq\left(\begin{array}{l}
1 \\
1 \\
1 \\
2 \\
2 \\
2 \\
2
\end{array}\right) .
\end{aligned}
$$

The set $\mathcal{G}(P)=\{(1,0,1,0,0,1),(0,1,0,1,0,1),(0,1,1,0,1,0),(1,0,0,1,1,0)\}$ contains its optima. It has formulation group $G_{P}=\left\langle\left(\begin{array}{lll}1 & 2\end{array}\right)\left(\begin{array}{lll}3 & 4\end{array}\right),\left(\begin{array}{ll}3 & 4\end{array}\right)\left(\begin{array}{ll}5 & 6\end{array}\right)\right\rangle$, which induces the orbits $\Omega_{G_{P}}=\left\{\omega_{1}, \omega_{2}, \omega_{3}\right\}=\{\{1,2\},\{3,4\},\{5,6\}\}$. It is easy to see that

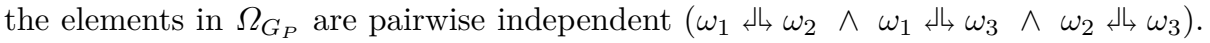


Consider for instance a restriction of the coefficient matrix to the columns indexed by orbits $\omega_{1}$ and $\omega_{2}$ :

$$
R=\left(\begin{array}{llll}
1 & 1 & 0 & 0 \\
0 & 0 & 1 & 1 \\
0 & 0 & 0 & 0 \\
1 & 0 & 1 & 0 \\
0 & 1 & 0 & 1 \\
1 & 0 & 0 & 1 \\
0 & 1 & 1 & 0
\end{array}\right)
$$

The action of permutation (1 2) on the columns of $R$ is equivalent to the action of permutation (1 4)(2 3) on the rows of $R$, both resulting in the following matrix:

$$
\left(\begin{array}{llll}
1 & 1 & 0 & 0 \\
0 & 0 & 1 & 1 \\
0 & 0 & 0 & 0 \\
0 & 1 & 1 & 0 \\
1 & 0 & 0 & 1 \\
0 & 1 & 0 & 1 \\
1 & 0 & 1 & 0
\end{array}\right) .
$$

Since mathematical programs are invariant under constraint-order permutations, we conclude that $\omega_{1}, 4 \omega_{2}$. The same argument is valid for the other cases. Nonetheless, $\omega_{1} \hookrightarrow\left\{\omega_{2}, \omega_{3}\right\}, \omega_{2} \hookrightarrow\left\{\omega_{1}, \omega_{3}\right\}$ and $\omega_{3} \hookrightarrow\left\{\omega_{1}, \omega_{2}\right\}$. In order to see this, we consider the full coefficient matrix (i.e. the three orbits simultaneously) and let the permutation (1 2) act on its columns; it yields the matrix:

$$
\left(\begin{array}{llllll}
1 & 1 & 0 & 0 & 0 & 0 \\
0 & 0 & 1 & 1 & 0 & 0 \\
0 & 0 & 0 & 0 & 1 & 1 \\
0 & 1 & 1 & 0 & 1 & 0 \\
1 & 0 & 0 & 1 & 1 & 0 \\
0 & 1 & 0 & 1 & 0 & 1 \\
1 & 0 & 1 & 0 & 0 & 1
\end{array}\right) .
$$

Note that in this case, there is no permutation of the rows of the coefficient matrix that produces the same matrix above, which means that we obtain a different set of constraints (i.e. a different mathematical program) when permuting the columns indexed by $\omega_{1}$ only. On the other hand, the action of $(12)(56)$ on the columns of the coefficient matrix is equivalent to the action of $(14)(23)$ on its rows, which means that we must permute the columns indexed by $\omega_{3}$ and $\omega_{1}$ simultaneously in order to obtain the original set of constraints; thus $\omega_{1} \hookrightarrow\left\{\omega_{2}, \omega_{3}\right\}$. The same argument is valid for the other cases.

\subsection{SBCs from independent sets}

Let $\Omega_{I}$ denote an independent set of orbits. Similarly to the results presented in [16], the following propositions set appropriate conditions to build weak and strong SBCs, respectively, from independent sets of orbtis. 
Proposition 1 The constraints (2) are $S B C s$ for $P$ and $G^{\Omega_{I}^{\omega}}$ with respect to $\omega \in \Omega_{I}$.

Proof Let $y \in \mathscr{G}(P)$. Since $G^{\Omega_{I}^{\omega}}$ acts transitively on $\omega$, there exists $\pi \in G^{\Omega_{I}^{\omega}}$ mapping $\min y[\omega]$ to $y_{\omega(1)}$.

Proposition 2 Provided that $G^{\Omega_{I}^{\omega}}[\omega]=\operatorname{Sym}(\omega)$, the constraints (1) are SBCs for $P$ and $G^{\Omega_{I}^{\omega}}$ with respect to $\omega \in \Omega_{I}$.

Proof Let $y \in \mathscr{G}(P)$. Since $G^{\Omega_{I}^{\omega}}[\omega]=\operatorname{Sym}(\omega)$, there exists $\pi \in G^{\Omega_{I}^{\omega}}$ such that $(\pi y)[\omega]$ is ordered by $\leq$. Thus $\pi y$ is feasible w.r.t. contraints (1).

\section{Orbital independence algorithm}

In this section we show how to solve the problem of finding an independent set of orbits of a mathematical program via a classical combinatorial optimization problem, and describe the algorithm proposed to build SBCs from this set. We also conclude our theoretical development by proving our main result concerning independent sets of orbits.

\subsection{Independence graph}

Our goal is to find the largest possible $\Omega_{I} \subseteq \Omega_{G_{P}}$. So far we do not have theoretical results providing sufficient conditions to find such a set. Yet we can use the necessary conditions provided by Corollary 1 and search for the largest set $\Omega_{K} \subseteq \Omega_{G_{p}}$ whose elements are pairwise independent. Having obtained $\Omega_{K}$, we can then search for the largest $\Omega_{I} \subseteq \Omega_{K}$. We propose to find $\Omega_{K}$ by solving the problem of finding the maximum clique in the (as of now called) independence graph of $P$.

Definition 7 The independence graph of $P$ is an undirected graph that encodes the independence relation between orbits of $G_{P}$, i.e., an undirected graph $G_{I}=(V, E)$ where $V=\Omega_{G_{P}}$ and $E$ is the set of pairwise independent ( 4 ) orbits of $\Omega_{G_{P}}$.

\subsection{OI reformulations}

We expect that the larger the number of SBCs adjoined to the original formulation, the stronger their computational impact. The larger the number of strong SBCs, the better. In fact it remains an open question what is the best trade-off in terms of computational impact: to add many weak, few strong or a mix of both SBCs to the same original formulation? In trying to shed some light on this matter, we propose two reformulations based on the concept of OI: the first prioritizing the total number of SBCs generated and the second prioritizing the total number of strong SBCs generated. In this sense, we look for cliques in $G_{I}$ that either involve large orbits or mostly orbits which may satisfy the conditions to build strong SBCs.

In order to find such cliques, we associate a weight function $w: V \rightarrow W$ to $G_{I}=(V, E, w)$ and solve the Maximum Weight Clique Problem (MWCP) for $G_{I}$ using the MP formulation described in [3]. In the first reformulation, which we call orbital independence narrowing, we have $W=\left\{\left|\omega_{1}\right|, \ldots,\left|\omega_{|V|}\right|\right\}$ and $w\left(\omega_{i}\right)=\left|\omega_{i}\right|$ for 
all $\omega_{i} \in V$. In the second, which we call strong orbital independence narrowing, we consider two different weight values $W=\left\{w_{1}, w_{2}\right\}$ with $w_{2}>w_{1}>0$, and assign $w_{1}$ to orbits which generate weak SBCs and $w_{2}$ to orbits which generate strong SBCs.

\subsection{Algorithm description}

The Algorithm 1 generates a system $C$ of compatible SBCs derived from the largest independent set of orbits of $P$. It takes as inputs a nontrivial formulation group (parameter $G_{P}$ ) and a reformulation strategy (parameter $\varsigma$ ). The following functions simplify the pseudocode of Alg. 1: computeOrbits $\left(G_{P}\right)$ returns the orbits of the group $G_{P}$; computePointStab $(\omega)$ returns the pointwise stabilizer of orbit $\omega$; $\operatorname{pos}(\omega)$ returns the position of orbit $\omega$ in the list $\Omega_{G_{P}}$; is Transitive $(G, \omega)$ returns true if the action of the group $G$ is transitive on the orbit $\omega$ and false otherwise; buildGraph $(V, E, \varsigma)$ returns a graph with vertices $V$, edges $E$ and weights appropriate to the strategy $\varsigma$; solveMWCP $\left(G_{I}\right)$ returns a solution of the MWCP for the graph $G_{I}$. We remark that the functions computeOrbits $\left(G_{P}\right)$, computePointStab $(\omega)$ and isTransitive $(G, \omega)$ are built-in functions available in the software package we use to carry out all grouprelated computations (see Section 5.3).

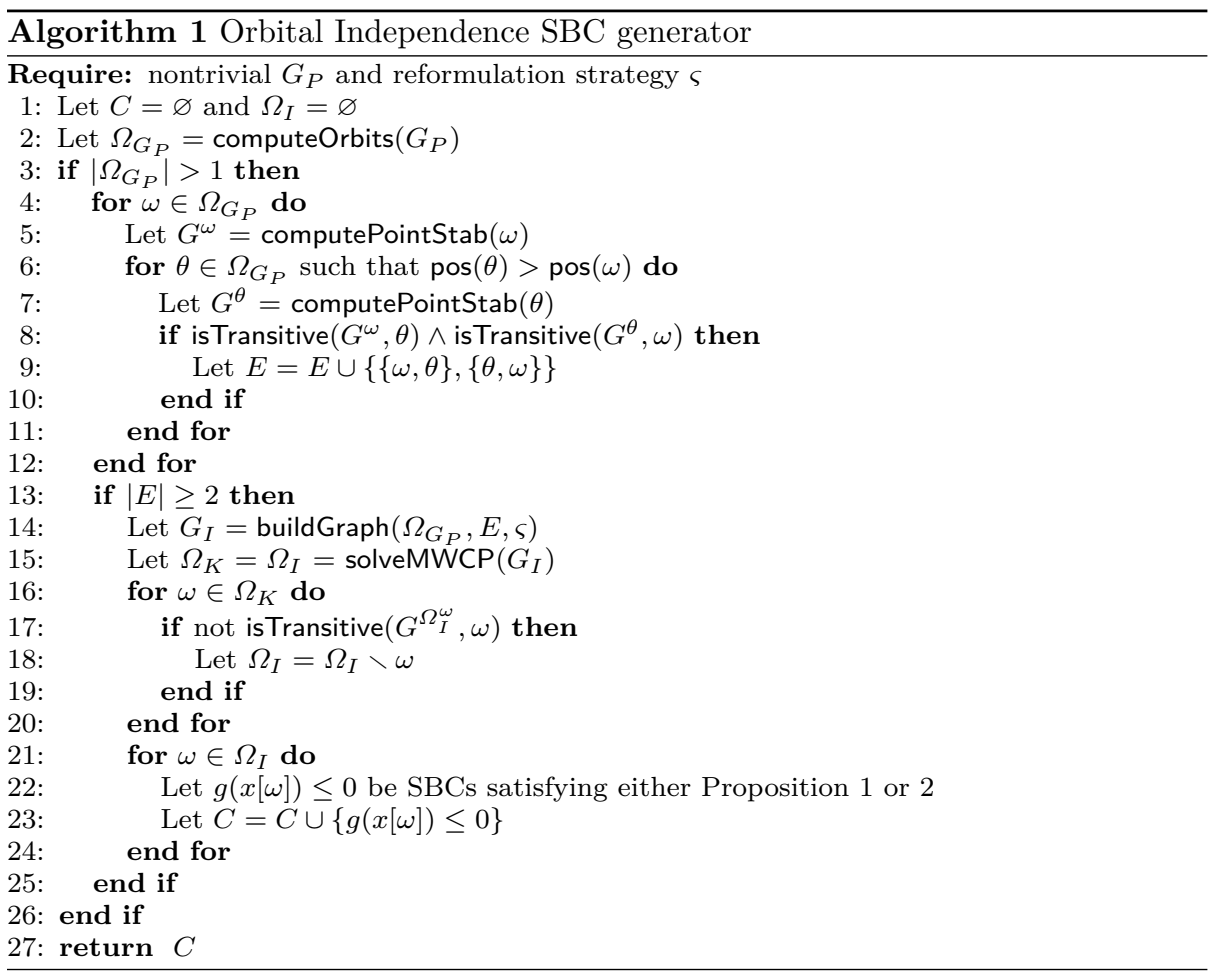

If $G_{P}$ has more than one orbit $\left(\left|\Omega_{G_{P}}\right|>1\right)$, the algorithm first iteratively looks for all the pairs of independent orbits to build the set $E$. Provided that the premise 
(3) in Theorem 1 is not sufficient to ascertain whether two orbits $\omega, \theta \in \Omega_{G_{P}}$ satisfy $\omega$, the algorithm does not compare the sets $\Gamma^{\omega}$ and $\Gamma^{\theta}$ but rather directly checks whether the action of the stabilizers $G^{\omega}$ and $G^{\theta}$ is transitive on $\theta$ and $\omega$, respectively. Testing transitivity is essential since it allows us to identify whether a set $X$ is an orbit of a group $G$ or not (see Section 2.1): in our context, if $G^{\omega}$ acts transitively on $\theta$, then $\theta\left\llcorner\omega\right.$ and if $G^{\theta}$ acts transitively on $\omega$, then $\omega\llcorner\theta$. In this case, we can add the edge $(\omega, \theta)$ to $E$ since $\omega$ ل

Following the first loop, if at least one pair of independent orbits is found $(|E| \geq$ 2 ), the algorithm builds the independence graph $G_{I}$ according to the reformulation strategy $\varsigma$ and calls a third party Mixed-Integer Linear Programming solver to solve the MWCP for $G_{I}$. Once $\Omega_{K}$ is known, the algorithm converges to a set $\Omega_{I}$ by iteratively removing (from a copy of $\Omega_{K}$ stored as $\Omega_{I}$ ) the orbits that do not satisfy $\omega \nrightarrow \Omega_{I}^{\omega}$.

Remark 3 Our approach here is not optimal since the resulting $\Omega_{I}$ may not be the largest one: evaluating all possible $\Omega_{I} \subseteq \Omega_{k}$ would require a huge computational effort owing to many stabilizer computations.

Then, for each orbit in $\Omega_{I}$, the algorithm builds and adds SBCs to the set $C$. It is relevant to emphasize that if $\left|\Omega_{G_{P}}\right|=1$ (unique orbit) or $|E|=0$ (no pair of independent orbits in $\Omega_{G_{P}}$ ), no reformulation is carried out.

Theorem 2 proves that any system of SBCs generated by Algorithm 1 for a given $G_{P}$ is a system of compatible SBCs for problem $P$, or in other words, it proves that independent sets of orbits embed sufficient conditions to exploit symmetries from two or more distinct orbits simultaneously.

Theorem 2 The constraint set $C_{\Omega_{I}}=\left\{g\left(x\left[\omega_{k}\right]\right) \leq 0 \mid \omega_{k} \in \Omega_{I}\right\}$ is a system of compatible SBCs for $P$.

Proof If $P$ is infeasible then adjoining the constraints in $C_{\Omega_{I}}$ to $P$ does not change its infeasibility, so assume $P$ is feasible. Since $g\left(x\left[\omega_{k}\right]\right) \leq 0$ are SBCs for $P$ and $G^{\Omega_{I}^{\omega_{k}}}$ with respect to $\omega_{k}$ (i.e. satisfying either Proposition 1 or 2 ), there exist $y \in \mathscr{G}(P)$ and $\pi_{\omega_{k}} \in G^{\Omega_{I}^{\omega_{k}}}$ such that $\pi_{\omega_{k}} y$ satisfies $g\left(\left(\pi_{\omega_{k}} y\right)\left[\omega_{k}\right]\right) \leq 0$. But $\pi_{\omega_{k}} \in G_{P}$ for all $\omega_{k} \in \Omega_{I}$ and, due to the closure of the group operation, there exists $\pi \in G_{P}$ such that $\pi=\prod \pi_{\omega_{k}}$. So $\pi y \in \mathscr{G}(P)$. But $\pi\left[\omega_{k}\right]=\pi_{\omega_{k}}\left[\omega_{k}\right]$ since $\pi_{\omega_{k^{\prime}}}$ stabilizes $\omega_{k}$ pointwise for every $k^{\prime} \neq k$ and thus $(\pi y)\left[\omega_{k}\right]=\left(\pi_{\omega_{k}} y\right)\left[\omega_{k}\right]$. Therefore $\pi y$ satisfies $g\left((\pi y)\left[\omega_{k}\right]\right) \leq 0$ for all $\omega_{k} \in \Omega_{I}$.

Finally, we would like to remark that the algorithms presented in [16] (coprime), [19] (stabilizer's chain) and Algorithm 1, with high probability, generate different sets of SBCs for the same mathematical program. The main reason for this is the fact that the coprime and the stabilizer's chain algorithms perform arbitrary orbit picks on every execution. As pointed out in Remark 2, for the same mathematical program, different runs of the same algorithm may result in different sets of SBCs. Moreover, for a given orbit, the order used to generate the SBCs is also randomly choosen by each algorithm, each choice leading to a different set of SBCs (see Remark 1). An exception would be the case where the stabilizer's chain algorithm luckily picks, at every iteration, the orbits that constitute the independent set found by Algorithm 1 (on top of the respective order for each orbit), what is unlike to happen as the number of orbits $\left|\Omega_{G_{P}}\right|$ increases. 


\section{Computational experiments}

In this section we show the computational impact on the resolution of symmetric $\mathbb{M I L P}$ s, MINLPS and $\mathbb{B} Q \mathbb{P P}_{s}$ when adjoining SBCs from independent sets of orbits. We describe the computational environment involved and analyze the results obtained from the conducted experiments.

\subsection{Symmetric BQP}

First we define the symmetric Binary Quadratic Programs used in our computational experiments. We are interested in $\mathbb{B} \mathbb{Q P S}$ in the form:

$$
\left.\begin{array}{cl}
\min _{x} & x^{\top} A_{0} x \\
\forall i \in \mathcal{I}_{E} & a_{i}^{\top} x=b_{i}, \\
& x \in\{0,1\}^{n} .
\end{array}\right\}
$$

where $A_{0}$ denotes a $n \times n$ real (possibly indefinite) symmetric matrix, $a_{i}$ denotes a vector of dimension $n$ for all $i \in \mathcal{I}_{E}, b$ denotes a vector of dimension $\left|\mathcal{I}_{E}\right|$ and $x$ represents a $n$ dimensional vector of binary decision variables.

Let 1 denote the $n$ dimensional all-ones vector. The first definition relates to the feasible region.

Definition 8 The feasible region has one single equality constraint of the type $\mathbf{1}^{\top} x=\lceil n / 2\rceil$.

Being invariant to permutations, the constraint in Definition 8 allows us to specify the structure of the formulation groups by controlling the strutucture of the matrix $A_{0}$ alone, which is defined next.

Definition $9 A_{0}$ is a block diagonal matrix.

Recall that the action of the formulation group on the index set defines a partition; and every member of the partition which has two or more indices is an orbit. We use this observation in our (quite simple) generation procedure: it first divides the indices of the decision variables into a partition $\mathcal{P}$, and then randomly decides whether each subset $s \in \mathcal{P}$ shall become an orbit or not (all according to some input data provided by the user). Each $s$ corresponds to a block in the matrix $A_{0}$. If $s$ is an orbit, the entries of the block $B_{s}$ are computed by sampling a pair $\left(z_{1}, z_{2}\right)$ of natural numbers and defining

$$
B_{s}=\left\{\begin{array}{cc}
z_{1}+(|s|-1) z_{2} & \text { if } i=j \\
-z_{2} & \text { if } i \neq j .
\end{array}\right.
$$

These blocks are Diagonal Dominant matrices. Otherwise ( $s$ is not an orbit), the entries of the block $B_{s}$ are computed by sampling a $(|s| \times|s|)$-matrix $M_{s}$ and defining

$$
B_{s}=M_{s}^{\top} M_{s} .
$$

These blocks are Gram matrices. Since all blocks of $A_{0}$ are Positive Semidefinite (PSD), the matrix $A_{0}$ is PSD as well and the continuous relaxations of the $\mathbb{B} \mathbb{Q P S}$ are convex. 
Definition 10 The blocks of $A_{0}$ are build according to Eq. (4) for orbits and according to Eq. (5) otherwise.

When the three definitions above are put together, they induce the following symmetry properties on the formulation group of the $\mathbb{B Q P P S}$ (a) $\Omega_{I}=\Omega_{G_{P}}$ and (b) $G_{P}[\omega]=\operatorname{Sym}(\omega)$ for every orbit $\omega \in \Omega_{G_{P}}$. These conditions allow us to concurrently use SBCs derived from all orbits of these $\mathbb{B Q P}$. Example 3 illustrates one of these programs.

Example 3 Let $P$ be the following $\mathbb{B} \mathbb{Q P}$ :

$$
\begin{array}{ll}
\min _{x \in\{0,1\}^{9}} & x^{\top} A_{0} x \\
& \mathbf{1}^{\top} x=5,
\end{array}
$$

where

$$
A_{0}=\left(\begin{array}{ccccccccc}
6 & -3 & -3 & 0 & 0 & 0 & 0 & 0 & 0 \\
-3 & 6 & -3 & 0 & 0 & 0 & 0 & 0 & 0 \\
-3 & -3 & 6 & 0 & 0 & 0 & 0 & 0 & 0 \\
0 & 0 & 0 & 12 & -6 & -6 & 0 & 0 & 0 \\
0 & 0 & 0 & -6 & 12 & -6 & 0 & 0 & 0 \\
0 & 0 & 0 & -6 & -6 & 12 & 0 & 0 & 0 \\
0 & 0 & 0 & 0 & 0 & 0 & 3 & 1 & 3 \\
0 & 0 & 0 & 0 & 0 & 0 & 1 & 5 & -2 \\
0 & 0 & 0 & 0 & 0 & 0 & 3 & -2 & 5
\end{array}\right)
$$

Since $n=9$, it is easy to see that it satisfies Def. $8:\lceil 9 / 2\rceil=5$. It is also clear that Def. 9 is satisfied since $A_{0}$ has a block diagonal shape. The indices are partioned into $\mathcal{P}=\{\{1,2,3\},\{4,5,6\},\{7,8,9\}\}$. The first two subsets $(\{1,2,3\},\{4,5,6\})$ are choosen to become orbits, and the pairs $(0,3)$ and $(0,6)$ are used to build the blocks associated to them, respectively. As concerns subset $\{7,8,9\}$, the matrix

$$
M_{s}=\left(\begin{array}{ccc}
1 & 1 & -1 \\
2 & 0 & 1 \\
0 & 1 & -2
\end{array}\right)
$$

is used to build its block. As a result, $\Omega_{G_{P}}=\left\{\omega_{1}, \omega_{2}\right\}=\{\{1,2,3\},\{4,5,6\}\}, \omega_{1}$ ل $\omega_{2}$ holds, and the transitive constituent of both orbits is isomorphic to $S_{3}$.

These BQP instances are particular cases of the Binary Quadratic Knapsack Problem (BQKP) [12] where (a) each orbit represents a set of identical objects, (b) all objects have the same size, (c) the knapsack has size $\lceil n / 2\rceil$, and (d) a pick within an orbit (variable set to 1) does not affect a pick within a different orbit cost-wise (because of the block-diagonal structure of matrix $A_{0}$ ). As an example, for $n=23$ and $\left|\Omega_{G_{P}}\right|=3$, orbit $\omega_{1}$ could represent ten apples, orbit $\omega_{2}$ seven oranges and orbit $\omega_{3}$ six pears; all apples, oranges and pears would have size one in our case, and the knapsack would have size $\lceil 23 / 2\rceil=12$. However, since we are not using any of the classical BQKP formulations, we prefer to present them simply as symmetric $\mathrm{BQP}$ instances.

Moreover, we could not find BQP instances in public libraries where Definitions 8, 9 and 10 occur simultaneously. Since we could not assume that such an application 
does not exist in practice, we designed these highly symmetric instances to at least provide guidelines and help readers to decide whether to employ or not the OI theory when solving the application they have in hands. By explicitly showcasing the symmetry properties under which the OI theory performs usefully, we highlight what sort of characteristics should be looked for in mathematical programs.

\subsection{Datasets}

Our test bed consists of three groups of instances: (a) symmetric $\mathbb{M I L P}$ s found in MIPLIB2010; (b) symmetric $\mathbb{M} I \mathbb{N} L \mathbb{P}$ s found in MINLPLib2 and (c) symmetric $\mathbb{B} \mathbb{Q} P_{S}$ generated via procedure described previously.

We found 89 instances within the public libraries, 47 from MIPLIB2010 and 42 from MINLPLib2. We refer to [26] for a detailed description of the presence of symmetries in public MP instances. The third group contains a total of 74 convex medium-sized $\mathbb{B} \mathbb{Q P}$, named as bqp_n_oxs, where $n$ represents the number of variables, $o$ the number of orbits and $s$ the orbits' size (R means random sizes).

\subsection{Environment}

The reformulations were obtained on a 4 -CPU Intel Xeon at $2.66 \mathrm{GHz}$ with $24 \mathrm{~Gb}$ RAM. Automatic group detection is carried out using ROSE [18] and TRACES [24]. Other group computations are carried out using GAP v. 4.7.4 [27]. The MP results were obtained on a $24-\mathrm{CPU}$ Intel Xeon at $2.53 \mathrm{GHz}$ with $48 \mathrm{~Gb}$ RAM. We used CPLEX 12.6 [13] to solve the MILPS and the $\mathbb{B} \mathbb{Q P S}$, and SCIP 3.0.1 [1] to solve the $\mathbb{M I N L} \mathbb{P}$, all under the AMPL [9] environment. The $\mathbb{B} \mathbb{Q P}$ generator was coded in Python 2.7.

The computation time was limited to 7200 seconds of user cpu time. In order to try and provide a fair assessment of our methodology, we disabled the symmetry handling methods built into CPLEX and ran it in single thread mode. SCIP does not contain internal symmetry handling methods.

\subsection{MILP and MINLP Results}

We first comment the results of the reformulation process. Tables 1 and 2 report, per instance, the number of variables $(n)$ and orbits $\left(\left|\Omega_{G_{P}}\right|\right)$ of the original formulation, and the number of variables indexed by orbits $\Omega_{G_{P}}$ (\#svar); for each OI narrowing type, they report the maximum clique $\left(\left|\Omega_{K}\right|\right)$ and the largest independent set $\left(\left|\Omega_{I}\right|\right)$ sizes, the number of variables indexed by orbits $\Omega_{I}$ (\#var), the number of weak (\#wea) and strong (\#str) SBCs, and the parameters $\sigma, \rho$ and $v$ (described later on).

\begin{tabular}{|c|c|c|c|c|c|c|c|c|c|c|c|}
\hline \multirow[b]{2}{*}{ Instance } & \multicolumn{3}{|c|}{ Original formulation } & \multicolumn{8}{|c|}{ OI-narrowing } \\
\hline & $n$ & $\left|\Omega_{G_{P}}\right|$ & \#svar & $\left|\Omega_{K}\right|$ & $\left|\Omega_{I}\right|$ & \#var & \#wea & \#str & $\sigma$ & $\rho$ & $v$ \\
\hline bab5 & 21600 & 1936 & 3872 & 4 & 4 & 8 & 0 & 4 & .17 & $0^{*}$ & $0^{*}$ \\
\hline blp-ar98 & 16017 & 2 & 4 & 2 & 2 & 4 & 0 & 2 & $0^{*}$ & 1.00 & 1.00 \\
\hline blp-ic97 & 8445 & 2 & 4 & 2 & 2 & 4 & 0 & 2 & $0 *$ & 1.00 & 1.00 \\
\hline core2536-691 & 15288 & 88 & 187 & 12 & 12 & 29 & 3 & 14 & .01 & .13 & .15 \\
\hline core $4872-1529$ & 24605 & 505 & 1046 & 46 & 46 & 96 & 0 & 50 & .04 & .09 & .09 \\
\hline gmu-35-40 & 842 & 40 & 111 & 4 & 4 & 13 & 0 & 9 & .13 & .10 & .11 \\
\hline
\end{tabular}




\begin{tabular}{|c|c|c|c|c|c|c|c|c|c|c|c|}
\hline gmu-35-50 & 1177 & 40 & 111 & 4 & 4 & 13 & 0 & 9 & .09 & .10 & .11 \\
\hline gmut-75-50 & 36164 & 64 & 242 & 6 & 6 & 19 & 0 & 13 & $0^{*}$ & .09 & .07 \\
\hline gmut-77-40 & 13140 & 70 & 280 & 7 & 7 & 26 & 0 & 19 & .02 & .10 & .09 \\
\hline iis-bupa-cov & 345 & 2 & 7 & 2 & 2 & 7 & 0 & 5 & .02 & 1.00 & 1.00 \\
\hline lectsched-4-obj & 3513 & 267 & 557 & 17 & 17 & 36 & 0 & 19 & .15 & .06 & .06 \\
\hline macrophage & 2260 & 251 & 566 & 18 & 18 & 42 & 5 & 19 & .25 & .07 & .07 \\
\hline map06 & 46015 & 107 & 245 & 10 & 10 & 20 & 0 & 10 & $0^{*}$ & .09 & .08 \\
\hline map10 & 46015 & 107 & 245 & 10 & 10 & 20 & 0 & 10 & $0^{*}$ & .09 & .08 \\
\hline map14 & 46015 & 107 & 245 & 10 & 10 & 20 & 0 & 10 & $0^{*}$ & .09 & .08 \\
\hline map18 & 46015 & 107 & 245 & 10 & 10 & 20 & 0 & 10 & $0^{*}$ & .09 & .08 \\
\hline map20 & 46015 & 107 & 245 & 10 & 10 & 20 & 0 & 10 & $0^{*}$ & .09 & .08 \\
\hline mcsched & 1669 & 45 & 90 & 15 & 15 & 30 & 0 & 15 & .05 & .33 & .33 \\
\hline mzzv11 & 10240 & 155 & 310 & 16 & 16 & 32 & 0 & 16 & .03 & .10 & .10 \\
\hline neos-1311124 & 1092 & 52 & 1092 & 4 & 4 & 84 & 0 & 80 & 1.00 & .07 & .07 \\
\hline neos-1426635 & 520 & 52 & 520 & 4 & 4 & 40 & 0 & 36 & 1.00 & .07 & .07 \\
\hline neos-1426662 & 832 & 52 & 832 & 4 & 4 & 64 & 0 & 60 & 1.00 & .07 & .07 \\
\hline neos-1436709 & 676 & 52 & 676 & 4 & 4 & 52 & 0 & 48 & 1.00 & .07 & .07 \\
\hline neos-1440460 & 468 & 52 & 468 & 4 & 4 & 36 & 0 & 32 & 1.00 & .07 & .07 \\
\hline neos-1442119 & 728 & 52 & 728 & 4 & 4 & 56 & 0 & 52 & 1.00 & .07 & .07 \\
\hline neos-1442657 & 624 & 52 & 624 & 4 & 4 & 48 & 0 & 44 & 1.00 & .07 & .07 \\
\hline neos-555424 & 3815 & 132 & 3810 & 8 & 8 & 190 & 107 & 75 & .99 & .06 & .04 \\
\hline neos-826841 & 5516 & 156 & 5436 & 3 & 3 & 200 & 191 & 6 & .98 & .01 & .03 \\
\hline neos- 849702 & 1737 & 128 & 1737 & 2 & 2 & 36 & 34 & 0 & 1.00 & .01 & .02 \\
\hline neos-911880 & 888 & 259 & 888 & 7 & 7 & 24 & 0 & 17 & 1.00 & .02 & .02 \\
\hline neos-952987 & 31329 & 37 & 81 & 4 & 4 & 8 & 0 & 4 & $0^{*}$ & .10 & .09 \\
\hline neos 18 & 963 & 53 & 248 & 5 & 5 & 26 & 0 & 21 & .25 & .09 & .10 \\
\hline ns1631475 & 22696 & 105 & 210 & 11 & 11 & 22 & 0 & 11 & $0^{*}$ & .10 & .10 \\
\hline ns2081729 & 661 & 300 & 600 & 3 & 3 & 6 & 0 & 3 & .90 & .01 & .01 \\
\hline p2m2p1m1p0n100 & 100 & 25 & 92 & 3 & 3 & 12 & 0 & 9 & .92 & .12 & .13 \\
\hline protfold & 1835 & 558 & 1800 & 2 & 2 & 4 & 0 & 2 & .98 & $0^{*}$ & $0^{*}$ \\
\hline rocII-4-11 & 3409 & 2 & 27 & 2 & 2 & 27 & 0 & 25 & $0^{*}$ & 1.00 & 1.00 \\
\hline rococoC10-001000 & 2566 & 41 & 82 & 4 & 4 & 8 & 0 & 4 & .03 & .09 & .09 \\
\hline rvb-sub & 33765 & 113 & 226 & 12 & 12 & 24 & 0 & 12 & $0^{*}$ & .10 & .10 \\
\hline satellites1-25 & 9013 & 200 & 400 & 20 & 20 & 40 & 0 & 20 & .04 & .10 & .10 \\
\hline seymour-disj-10 & 1209 & 49 & 106 & 5 & 5 & 12 & 0 & 7 & .08 & .10 & .11 \\
\hline seymour & 1255 & 55 & 156 & 5 & 5 & 41 & 29 & 7 & .12 & .09 & .26 \\
\hline swath & 6404 & 21 & 163 & 2 & 2 & 8 & 0 & 6 & .02 & .09 & .04 \\
\hline transportmoment & 9099 & 85 & 189 & 17 & 17 & 38 & 0 & 21 & .02 & .20 & .20 \\
\hline toll-like & 2883 & 386 & 1091 & 26 & 26 & 91 & 44 & 21 & .37 & .06 & .08 \\
\hline uc-case 3 & 36921 & 2687 & 5374 & 2 & 2 & 4 & 0 & 2 & .14 & $0^{*}$ & $0^{*}$ \\
\hline uct-subprob & 2236 & 136 & 306 & 7 & 7 & 14 & 0 & 7 & .13 & .05 & .04 \\
\hline & \multicolumn{3}{|c|}{ Original formulation } & \multicolumn{8}{|c|}{ SOI-narrowing } \\
\hline Instance & $n$ & $\left|\Omega_{G_{P}}\right|$ & \#svar & $\left|\Omega_{K}\right|$ & $\left|\Omega_{I}\right|$ & \#var & \#wea & \#str & $\sigma$ & $\rho$ & $v$ \\
\hline core2536-691 & 15288 & 88 & 187 & 12 & 12 & 27 & 0 & 15 & .01 & .13 & .14 \\
\hline macrophage & 2260 & 251 & 566 & 18 & 18 & 39 & 0 & 21 & .25 & .07 & .06 \\
\hline neos-555424 & 3815 & 132 & 3810 & 8 & 8 & 145 & 58 & 79 & .99 & .06 & .03 \\
\hline neos- 826841 & 5516 & 156 & 5436 & 4 & 4 & 46 & 0 & 42 & .98 & .02 & $0^{*}$ \\
\hline neos-849702 & 1737 & 128 & 1737 & 2 & 2 & 9 & 0 & 7 & 1.00 & .01 & $0^{*}$ \\
\hline toll-like & 2883 & 386 & 1091 & 26 & 26 & 59 & 0 & 33 & .37 & .06 & .05 \\
\hline
\end{tabular}

Table 1: OI-narrowings of symmetric instances from MIPLIB2010. $0^{*}$ indicates values of $O\left(10^{-3}\right)$ or less.

\begin{tabular}{|c|c|c|c|c|c|c|c|c|c|c|c|}
\hline & \multicolumn{3}{|c|}{ Original formulation } & \multicolumn{8}{|c|}{ OI-narrowing } \\
\hline Instance & $n$ & $\left|\Omega_{G_{P}}\right|$ & \#svar & $\left|\Omega_{K}\right|$ & $\left|\Omega_{I}\right|$ & \#var & \#wea & \#str & $\sigma$ & $\rho$ & $v$ \\
\hline arki0002 & 2456 & 384 & 2304 & 2 & 2 & 12 & 0 & 10 & .93 & $0^{*}$ & $0^{*}$ \\
\hline arki0005 & 2370 & 9 & 18 & 9 & 9 & 18 & 0 & 9 & $0^{*}$ & 1.00 & 1.00 \\
\hline arki0006 & 2370 & 9 & 18 & 9 & 9 & 18 & 0 & 9 & $0^{*}$ & 1.00 & 1.00 \\
\hline autocorr_bern25-03 & 26 & 12 & 24 & 2 & 2 & 4 & 0 & 2 & .92 & 16 & .16 \\
\hline carton 7 & 230 & 49 & 162 & 3 & 3 & 13 & 8 & 2 & .70 & .06 & .08 \\
\hline carton 9 & 266 & 83 & 266 & 3 & 3 & 13 & 8 & 2 & 1.00 & .03 & .04 \\
\hline cecil_13 & 733 & 18 & 36 & 9 & 9 & 18 & 0 & 9 & .04 & .50 & .50 \\
\hline chp_partload & 2080 & 82 & 164 & 5 & 5 & 10 & 0 & 5 & .07 & .06 & .06 \\
\hline crudeoil_li21 & 1236 & 134 & 268 & 2 & 2 & 4 & 0 & 2 & .21 & .01 & .01 \\
\hline ex9_2_6 & 16 & 7 & 16 & 2 & 2 & 6 & 3 & 1 & 1.00 & .28 & .37 \\
\hline gastrans & 89 & 6 & 12 & 2 & 2 & 4 & 0 & 2 & .13 & .33 & .33 \\
\hline hmittelman & 16 & 3 & 6 & 3 & 3 & 6 & 0 & 3 & .37 & 1.00 & 1.00 \\
\hline kport20 & 98 & 25 & 55 & 5 & 5 & 11 & 0 & 6 & .56 & 20 & .20 \\
\hline kport40 & 217 & 48 & 150 & 8 & 8 & 28 & 0 & 20 & .69 & .16 & .18 \\
\hline lop97ic & 1626 & 3 & 127 & 3 & 3 & 127 & 0 & 124 & .07 & 1.00 & 1.00 \\
\hline lop97icx & 986 & 8 & 777 & 8 & 8 & 777 & 0 & 769 & .78 & 1.00 & 1.00 \\
\hline mbtd & 210 & 61 & 210 & 2 & 2 & 12 & 9 & 1 & 1.00 & .03 & .05 \\
\hline
\end{tabular}




\begin{tabular}{|c|c|c|c|c|c|c|c|c|c|c|c|}
\hline netmod_kar1 & 456 & 48 & 132 & 3 & 3 & 9 & 0 & 6 & .28 & .06 & .06 \\
\hline netmod_kar2 & 456 & 48 & 132 & 3 & 3 & 9 & 0 & 6 & .28 & .06 & .06 \\
\hline powerflow $2383 \mathrm{wpr}$ & 15882 & 12 & 24 & 3 & 3 & 6 & 0 & 3 & $0^{*}$ & .25 & .25 \\
\hline powerflow $2383 \mathrm{wpp}$ & 15882 & 12 & 24 & 3 & 3 & 6 & 0 & 3 & $0^{*}$ & .25 & .25 \\
\hline risk $2 \mathrm{bpb}$ & 434 & 12 & 72 & 12 & 12 & 72 & 0 & 60 & .16 & 1.00 & 1.00 \\
\hline routingdelay_bigm & 1115 & 18 & 36 & 12 & 12 & 24 & 0 & 12 & .03 & .66 & .66 \\
\hline routingdelay_proj & 1115 & 18 & 36 & 12 & 12 & 24 & 0 & 12 & .03 & .66 & .66 \\
\hline sepasequ_complex & 485 & 5 & 27 & 5 & 5 & 27 & 9 & 13 & .05 & 1.00 & 1.00 \\
\hline st_rv9 & 50 & 10 & 20 & 10 & 10 & 20 & 0 & 10 & .40 & 1.00 & 1.00 \\
\hline super 1 & 1263 & 12 & 26 & 12 & 12 & 26 & 0 & 14 & .02 & 1.00 & 1.00 \\
\hline super2 & 1274 & 11 & 24 & 11 & 11 & 24 & 0 & 13 & .01 & 1.00 & 1.00 \\
\hline super3 & 1281 & 11 & 24 & 11 & 11 & 24 & 0 & 13 & .01 & 1.00 & 1.00 \\
\hline super3t & 1032 & 11 & 24 & 11 & 11 & 24 & 0 & 13 & .02 & 1.00 & 1.00 \\
\hline syn $15 \mathrm{~m}$ & 55 & 2 & 5 & 2 & 2 & 5 & 0 & 3 & .09 & 1.00 & 1.00 \\
\hline torsion 100 & 5004 & 2 & 4 & 2 & 2 & 4 & 0 & 2 & $0^{*}$ & 1.00 & 1.00 \\
\hline torsion 25 & 1254 & 2 & 4 & 2 & 2 & 4 & 0 & 2 & $0 *$ & 1.00 & 1.00 \\
\hline torsion 50 & 2504 & 1227 & 2454 & 3 & 3 & 6 & 0 & 3 & .98 & $0^{*}$ & $0^{*}$ \\
\hline torsion 75 & 3754 & 2 & 4 & 2 & 2 & 4 & 0 & 2 & $0^{*}$ & 1.00 & 1.00 \\
\hline transswitch $2383 \mathrm{wpr}$ & 18768 & 15 & 30 & 3 & 3 & 6 & 0 & 3 & $0^{*}$ & .20 & .20 \\
\hline transswitch 2383 wpp & 18768 & 15 & 30 & 3 & 3 & 6 & 0 & 3 & $0^{*}$ & .20 & .20 \\
\hline turkey & 512 & 4 & 8 & 4 & 4 & 8 & 0 & 4 & .01 & 1.00 & 1.00 \\
\hline unitcommit 1 & 738 & 2 & 30 & 2 & 2 & 30 & 0 & 28 & .04 & 1.00 & 1.00 \\
\hline unitcommit2 & 738 & 2 & 30 & 2 & 2 & 30 & 0 & 28 & .04 & 1.00 & 1.00 \\
\hline waste & 1425 & 30 & 76 & 15 & 15 & 38 & 0 & 23 & .05 & .50 & .50 \\
\hline waterund 28 & 760 & 106 & 216 & 2 & 2 & 4 & 0 & 2 & .28 & .01 & .01 \\
\hline & \multicolumn{3}{|c|}{ Original formulation } & \multicolumn{8}{|c|}{ SOI-narrowing } \\
\hline Instance & $n$ & $\left|\Omega_{G_{P}}\right|$ & \#svar & $\left|\Omega_{K}\right|$ & $\left|\Omega_{I}\right|$ & \#var & \#wea & \#str & $\sigma$ & $\rho$ & $v$ \\
\hline carton7 & 230 & 49 & 162 & 3 & 3 & 8 & $\overline{0}$ & 5 & .70 & $\overline{c .06}$ & $\overline{.04}$ \\
\hline carton 9 & 266 & 83 & 266 & 3 & 3 & 8 & 0 & 5 & 1.00 & .03 & .03 \\
\hline
\end{tabular}

Table 2: OI-narrowings of symmetric instances from MINLPLib2. $0 *$ indicates values of $O\left(10^{-3}\right)$ or less.

Both reformulation strategies yielded the same narrowings for the most part of the instances. In these cases, we do not present results concerning the SOI reformulation. Recall that Algorithm 1 can yield suboptimal independent sets in terms of size (see Remark 3 in Sect. 4.3). The reformulation results show that the size of the maximum cliques is equal to the size of the largest independent sets for all instances; we thus judge that Algorithm 1 yields good results on average as concerns symmetry detection.

\begin{tabular}{|c||cc|cc|cc|}
\hline \multicolumn{1}{|c|}{} & \multicolumn{2}{c|}{ Original formulation } & \multicolumn{2}{c|}{ OI-narrowing } & \multicolumn{2}{c|}{ SOI-narrowing } \\
\cline { 2 - 8 } & \# Best & Time $(\mathrm{h})$ & \# Best & Time $(\mathrm{h})$ & \# Best & Time $(\mathrm{h})$ \\
\hline \hline DIPLIB2010 & 22 & 49.52 & 20 & 48.16 & 4 & 48.15 \\
MINLPLib2 & 14 & 52.00 & 17 & 51.3 & 2 & 51.29 \\
\hline Total & 36 & 101.52 & 37 & 99.46 & 6 & 99.44 \\
\hline
\end{tabular}

Table 3: Aggregated solution statistics for datasets MIPLIB2010 and MINLPLib2.

Table 3 provides aggregated solution statistics. Per dataset and for each formulation, the table reports the number of best performances and the total time comsumed in hours to solve all instances. The statistics are more expressive regarding the MIPLIB2010 library.

Finally, Tables 4 and 5 report details of the optimization results. Per instance and for each formulation, the table exhibits the best solution found, the user cpu time (in seconds), the gap (\%), the number of $\mathrm{BB}$ nodes and the solver status at termination (opt $=$ optimum found, $\lim =$ time limit reached, inf $=$ infeasible instance $)$. Best 
values are emphasized in boldface. Two intances (namely powerflow2383wpp and transswitch2383wpp) do not appear in Table 5 due to SCIP technical limitations.

We observe that the total computation time of the OI-narrowings is 2 hours inferior (Table 3), which means that we improved overall, despite the factors that play against us (as explained below). Instance-wise the results may not be significant in some cases, but this goes both ways (original formulation vs oi-narrowings): the SBCs slightly helped to improve the performance of the solvers in 43 cases and were detrimental in 36 cases out of 87 . Despite of providing good results, the SOInarrowings did not achieve outstanding performances.

Our investigation indicates that strong OI reformulations occur seldomly in practice (it was found in $9 \%$ of the symmetric public instances tested), yet we encourage its use since the computational experiments also show that such narrowings helped to improve the solver's performance in $75 \%$ of the cases ( 6 out of 8 instances). This is a fairly good percentual when compared to the overall performance of the OI reformulations.

Overall, we think that two facts contribute to explain the average-to-poor computational results we have achieved with the public instances. First, apart from the structure of the group $G_{P}$, the ratio $\sigma=(\#$ svar $/ n)$ may also indicate how symmetric a formulation $P$ is. Similarly, the ratios $\rho=\left(\left|\Omega_{I}\right| /\left|\Omega_{G_{P}}\right|\right)$ and $v=(\#$ var/\#svar) may indicate how extensively one has exploited the symmetries of $P$. All together, we expect SBCs to make a strong computational impact whenever the triplet $(\sigma, \rho, v)$ tends to $(1,1,1)$. However, Tables 1 and 2 show the two patterns in which the majority of the instances fit into: either the instance is highly symmetric $(\sigma \approx 1)$ and we cannot explore much of its symmetries $(\rho, v) \approx(0,0)$, or it does not exhibit many symmetries $(\sigma \approx 0)$ and we explore almost all of them $(\rho, v) \approx(1,1)$. Second, recall that BB type algorithms are complex systems whose performance depend on many factors (Linear Programming (LP) solutions, branching policies, cut generation schemes and so on). The presence of SBCs may change LP solutions computed in the nodes of the BB tree, which means that SBCs can also unduly impact on branching policies and on cut generation schemes. Since there are elements of arbitrary choice regarding the generation of SBCs (recall Remarks 1 and 2), forcing these choices may, in some cases, prevent the $\mathrm{BB}$ algorithm to take the correct decisions.

\subsection{BQP Results}

Again we start off commenting the results related to the OI reformulation process. As the content of Table 6 indicates, the $\mathbb{B} \mathbb{Q P}$ s are highly symmetric. We observe that $(\sigma, \rho, v)=([0.5,1], 1,1)$ holds for all cases. Moreover, per $\mathbb{B} \mathbb{Q P}$ generated, every orbit satisfies the conditions in Proposition 2 and thus we could build nothing but strong SBCs for all instances.

\begin{tabular}{|c|c|c|c|c|c|c|c|c|c|c|c|}
\hline \multirow[b]{2}{*}{ Instance } & \multicolumn{3}{|c|}{ Original formulation } & \multicolumn{8}{|c|}{ OI-narrowing } \\
\hline & $n$ & $\left|\Omega_{G_{P}}\right|$ & \#svar & $\left|\Omega_{K}\right|$ & $\left|\Omega_{I}\right|$ & \#var & \#wea & \#str & $\sigma$ & $\rho$ & $v$ \\
\hline bqp_70_2xR & 70 & 2 & 49 & 2 & 2 & 49 & 0 & 47 & .70 & 1.00 & 1.00 \\
\hline bqp_70_3xR & 70 & 3 & 45 & 3 & 3 & 45 & 0 & 42 & .64 & 1.00 & 1.00 \\
\hline bqp_70_4x14 & 70 & 4 & 56 & 4 & 4 & 56 & 0 & 52 & .80 & 1.00 & 1.00 \\
\hline bqp_70_4xR & 70 & 4 & 70 & 4 & 4 & 70 & 0 & 66 & 1.00 & 1.00 & 1.00 \\
\hline bqp_70_5xR & 70 & 5 & 58 & 5 & 5 & 58 & 0 & 53 & .82 & 1.00 & 1.00 \\
\hline bqp_70_6x10 & 70 & 6 & 60 & 6 & 6 & 60 & 0 & 54 & .85 & 1.00 & 1.00 \\
\hline bqp_70_7xR & 70 & 7 & 63 & 7 & 7 & 63 & 0 & 56 & .90 & 1.00 & 1.00 \\
\hline bqp_70_9x7 & 70 & 9 & 63 & 9 & 9 & 63 & 0 & 54 & .90 & 1.00 & 1.00 \\
\hline
\end{tabular}




\begin{tabular}{|c|c|c|c|c|c|c|c|c|c|c|c|}
\hline bqp_75_2x 25 & 75 & 2 & 50 & 2 & 2 & 50 & 0 & 48 & .66 & 1.00 & 1.00 \\
\hline bqp_75_2xR & 75 & 2 & 39 & 2 & 2 & 39 & 0 & 37 & .52 & 1.00 & 1.00 \\
\hline bqp_75_3xR & 75 & 3 & 60 & 3 & 3 & 60 & 0 & 57 & .80 & 1.00 & 1.00 \\
\hline bqp_75_4x 15 & 75 & 4 & 60 & 4 & 4 & 60 & 0 & 56 & .80 & 1.00 & 1.00 \\
\hline bqp_75_4xR & 75 & 4 & 54 & 4 & 4 & 54 & 0 & 50 & .72 & 1.00 & 1.00 \\
\hline bqp_75_5x15 & 75 & 5 & 75 & 5 & 5 & 75 & 0 & 70 & 1.00 & 1.00 & 1.00 \\
\hline bqp_75_5xR & 75 & 5 & 66 & 5 & 5 & 66 & 0 & 61 & .88 & 1.00 & 1.00 \\
\hline bqp_75_6xR & 75 & 6 & 67 & 6 & 6 & 67 & 0 & 61 & .89 & 1.00 & 1.00 \\
\hline bqp_75_7xR & 75 & 7 & 63 & 7 & 7 & 63 & 0 & 56 & .84 & 1.00 & 1.00 \\
\hline bqp_75_8xR & 75 & 8 & 66 & 8 & 8 & 66 & 0 & 58 & .88 & 1.00 & 1.00 \\
\hline bqp_80_2x 20 & 80 & 2 & 40 & 2 & 2 & 40 & 0 & 38 & .50 & 1.00 & 1.00 \\
\hline bqp_80_2xR & 80 & 2 & 55 & 2 & 2 & 55 & 0 & 53 & .68 & 1.00 & 1.00 \\
\hline bqp_80_3x20 & 80 & 3 & 60 & 3 & 3 & 60 & 0 & 57 & .75 & 1.00 & 1.00 \\
\hline bqp_80_3xR & 80 & 3 & 64 & 3 & 3 & 64 & 0 & 61 & .80 & 1.00 & 1.00 \\
\hline bqp_80_4x16 & 80 & 4 & 64 & 4 & 4 & 64 & 0 & 60 & .80 & 1.00 & 1.00 \\
\hline bqp_80_4xR & 80 & 4 & 65 & 4 & 4 & 65 & 0 & 61 & .81 & 1.00 & 1.00 \\
\hline bqp_80_5x16 & 80 & 5 & 80 & 5 & 5 & 80 & 0 & 75 & 1.00 & 1.00 & 1.00 \\
\hline bqp_80_5xR & 80 & 5 & 70 & 5 & 5 & 70 & 0 & 65 & .87 & 1.00 & 1.00 \\
\hline bqp_80_6xR & 80 & 6 & 72 & 6 & 6 & 72 & 0 & 66 & .90 & 1.00 & 1.00 \\
\hline bqp_80_7 $7 \times 10$ & 80 & 7 & 70 & 7 & 7 & 70 & 0 & 63 & .87 & 1.00 & 1.00 \\
\hline bqp_80_8xR & 80 & 8 & 68 & 8 & 8 & 68 & 0 & 60 & .85 & 1.00 & 1.00 \\
\hline bqp_85_12x5 & 85 & 12 & 60 & 12 & 12 & 60 & 0 & 48 & .70 & 1.00 & 1.00 \\
\hline bqp_85_16x5 & 85 & 16 & 80 & 16 & 16 & 80 & 0 & 64 & .94 & 1.00 & 1.00 \\
\hline bqp_85_2x17 & 85 & 2 & 34 & 2 & 2 & 34 & 0 & 32 & .40 & 1.00 & 1.00 \\
\hline bqp_85_2xR & 85 & 2 & 59 & 2 & 2 & 59 & 0 & 57 & .69 & 1.00 & 1.00 \\
\hline bqp_85_3xR & 85 & 3 & 68 & 3 & 3 & 68 & 0 & 65 & .80 & 1.00 & 1.00 \\
\hline bqp_85_4x 17 & 85 & 4 & 68 & 4 & 4 & 68 & 0 & 64 & .80 & 1.00 & 1.00 \\
\hline bqp_85_4xR & 85 & 4 & 67 & 4 & 4 & 67 & 0 & 63 & .78 & 1.00 & 1.00 \\
\hline bqp_85_5xR & 85 & 5 & 64 & 5 & 5 & 64 & 0 & 59 & .75 & 1.00 & 1.00 \\
\hline bqp_85_6xR & 85 & 6 & 75 & 6 & 6 & 75 & 0 & 69 & .88 & 1.00 & 1.00 \\
\hline bqp_85_7xR & 85 & 7 & 76 & 7 & 7 & 76 & 0 & 69 & .89 & 1.00 & 1.00 \\
\hline bqp_85_8xR & 85 & 8 & 80 & 8 & 8 & 80 & 0 & 72 & .94 & 1.00 & 1.00 \\
\hline bqp_85_9xR & 85 & 9 & 85 & 9 & 9 & 85 & 0 & 76 & 1.00 & 1.00 & 1.00 \\
\hline bqp_90_2x30 & 90 & 2 & 60 & 2 & 2 & 60 & 0 & 58 & .66 & 1.00 & 1.00 \\
\hline bqp_90_2xR & 90 & 2 & 65 & 2 & 2 & 65 & 0 & 63 & .72 & 1.00 & 1.00 \\
\hline bqp_90_3 $3 \times 30$ & 90 & 3 & 90 & 3 & 3 & 90 & 0 & 87 & 1.00 & 1.00 & 1.00 \\
\hline bqp_90_3xR & 90 & 3 & 75 & 3 & 3 & 75 & 0 & 72 & .83 & 1.00 & 1.00 \\
\hline bqp_90_4x18 & 90 & 4 & 72 & 4 & 4 & 72 & 0 & 68 & .80 & 1.00 & 1.00 \\
\hline bqp_90_4xR & 90 & 4 & 73 & 4 & 4 & 73 & 0 & 69 & .81 & 1.00 & 1.00 \\
\hline bqp_90_5x15 & 90 & 5 & 75 & 5 & 5 & 75 & 0 & 70 & .83 & 1.00 & 1.00 \\
\hline bqp_90_5xR & 90 & 5 & 77 & 5 & 5 & 77 & 0 & 72 & .85 & 1.00 & 1.00 \\
\hline bqp_90_6xR & 90 & 6 & 76 & 6 & 6 & 76 & 0 & 70 & .84 & 1.00 & 1.00 \\
\hline bqp_90_7xR & 90 & 7 & 70 & 7 & 7 & 70 & 0 & 63 & .77 & 1.00 & 1.00 \\
\hline bqp_90_8x 10 & 90 & 8 & 80 & 8 & 8 & 80 & 0 & 72 & .88 & 1.00 & 1.00 \\
\hline bqp_90_9x9 & 90 & 9 & 81 & 9 & 9 & 81 & 0 & 72 & .90 & 1.00 & 1.00 \\
\hline bqp_95_18x5 & 95 & 18 & 90 & 18 & 18 & 90 & 0 & 72 & .94 & 1.00 & 1.00 \\
\hline bqp_95_2xR & 95 & 2 & 51 & 2 & 2 & 51 & 0 & 49 & .53 & 1.00 & 1.00 \\
\hline bqp_95_3xR & 95 & 3 & 77 & 3 & 3 & 77 & 0 & 74 & .81 & 1.00 & 1.00 \\
\hline bqp_95_4x19 & 95 & 4 & 76 & 4 & 4 & 76 & 0 & 72 & .80 & 1.00 & 1.00 \\
\hline bqp_95_4xR & 95 & 4 & 90 & 4 & 4 & 90 & 0 & 86 & .94 & 1.00 & 1.00 \\
\hline bqp_95_5xR & 95 & 5 & 88 & 5 & 5 & 88 & 0 & 83 & .92 & 1.00 & 1.00 \\
\hline bqp_95_6xR & 95 & 6 & 89 & 6 & 6 & 89 & 0 & 83 & .93 & 1.00 & 1.00 \\
\hline bqp_95_7xR & 95 & 7 & 95 & 7 & 7 & 95 & 0 & 88 & 1.00 & 1.00 & 1.00 \\
\hline bqp_95_8xR & 95 & 8 & 95 & 8 & 8 & 95 & 0 & 87 & 1.00 & 1.00 & 1.00 \\
\hline bqp_95_9xR & 95 & 9 & 86 & 9 & 9 & 86 & 0 & 77 & .90 & 1.00 & 1.00 \\
\hline bqp_100_2xR & 100 & 2 & 70 & 2 & 2 & 70 & 0 & 68 & .70 & 1.00 & 1.00 \\
\hline bqp_100_3 325 & 100 & 3 & 75 & 3 & 3 & 75 & 0 & 72 & .75 & 1.00 & 1.00 \\
\hline bqp_100_3xR & 100 & 3 & 77 & 3 & 3 & 77 & 0 & 74 & .77 & 1.00 & 1.00 \\
\hline bqp_100_4 $\times 20$ & 100 & 4 & 80 & 4 & 4 & 80 & 0 & 76 & .80 & 1.00 & 1.00 \\
\hline bqp_100_4xR & 100 & 4 & 81 & 4 & 4 & 81 & 0 & 77 & .81 & 1.00 & 1.00 \\
\hline bqp_100_5x20 & 100 & 5 & 100 & 5 & 5 & 100 & 0 & 95 & 1.00 & 1.00 & 1.00 \\
\hline bqp_100_5xR & 100 & 5 & 93 & 5 & 5 & 93 & 0 & 88 & .93 & 1.00 & 1.00 \\
\hline bqp_100_6xR & 100 & 6 & 96 & 6 & 6 & 96 & 0 & 90 & .96 & 1.00 & 1.00 \\
\hline bqp_100_7xR & 100 & 7 & 88 & 7 & 7 & 88 & 0 & 81 & .88 & 1.00 & 1.00 \\
\hline bqp_100_8xR & 100 & 8 & 89 & 8 & 8 & 89 & 0 & 81 & .89 & 1.00 & 1.00 \\
\hline bqp_100_9x10 & 100 & 9 & 90 & 9 & 9 & 90 & 0 & 81 & .90 & 1.00 & 1.00 \\
\hline
\end{tabular}

Table 6: OI narrowings of symmetric $\mathbb{B} \mathbb{Q P S}$. $0^{*}$ indicates values of $O\left(10^{-3}\right)$ or less.

Table 7 presents the aggregated statistics for the BQP dataset. In the majority of the cases, 51 out of 74, the narrowings performed better, against 21 of the original formulations. Note however the large difference in terms of execution time: more than 11 hours in total for the original problems against less than 17 seconds for 

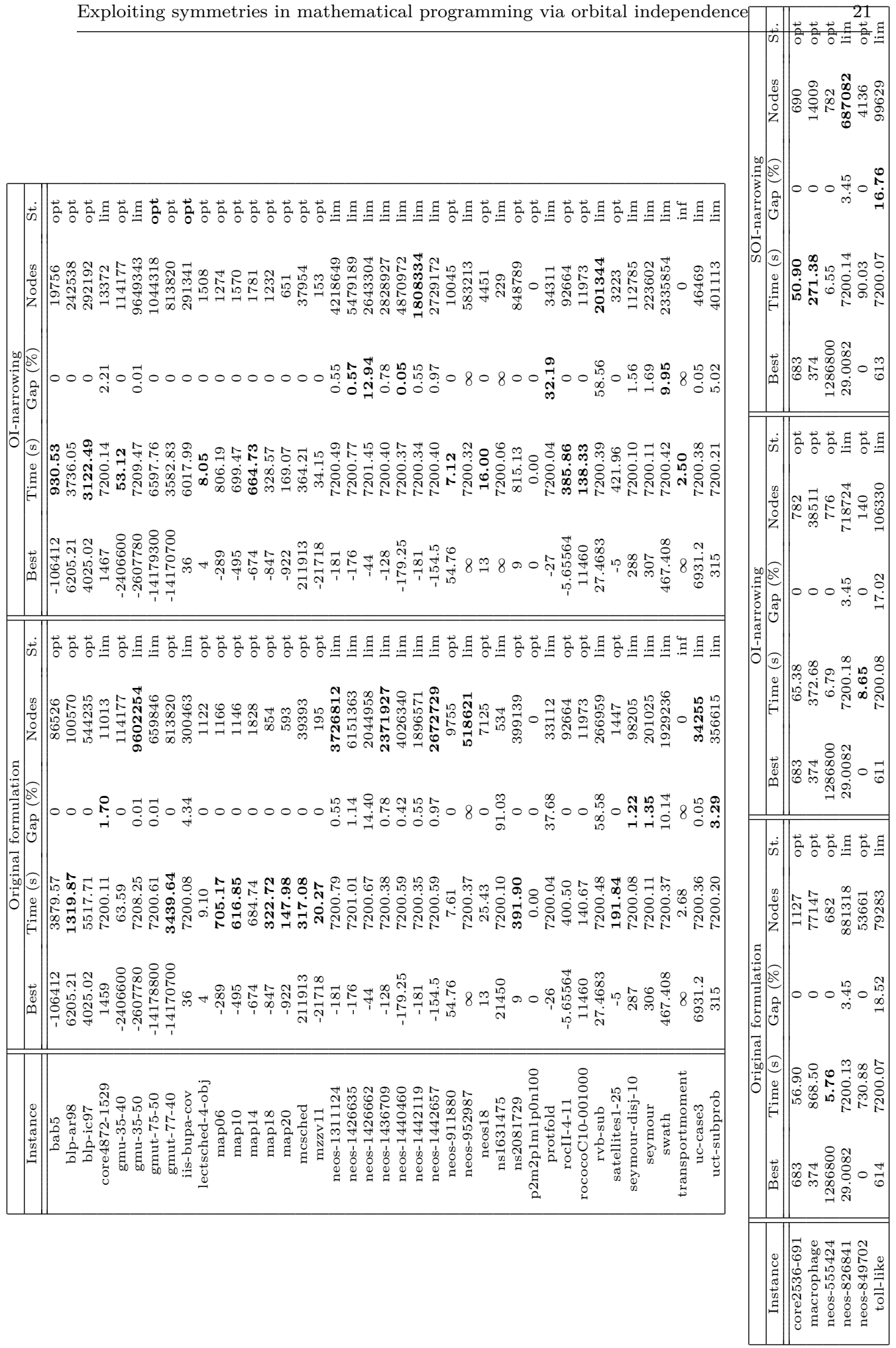


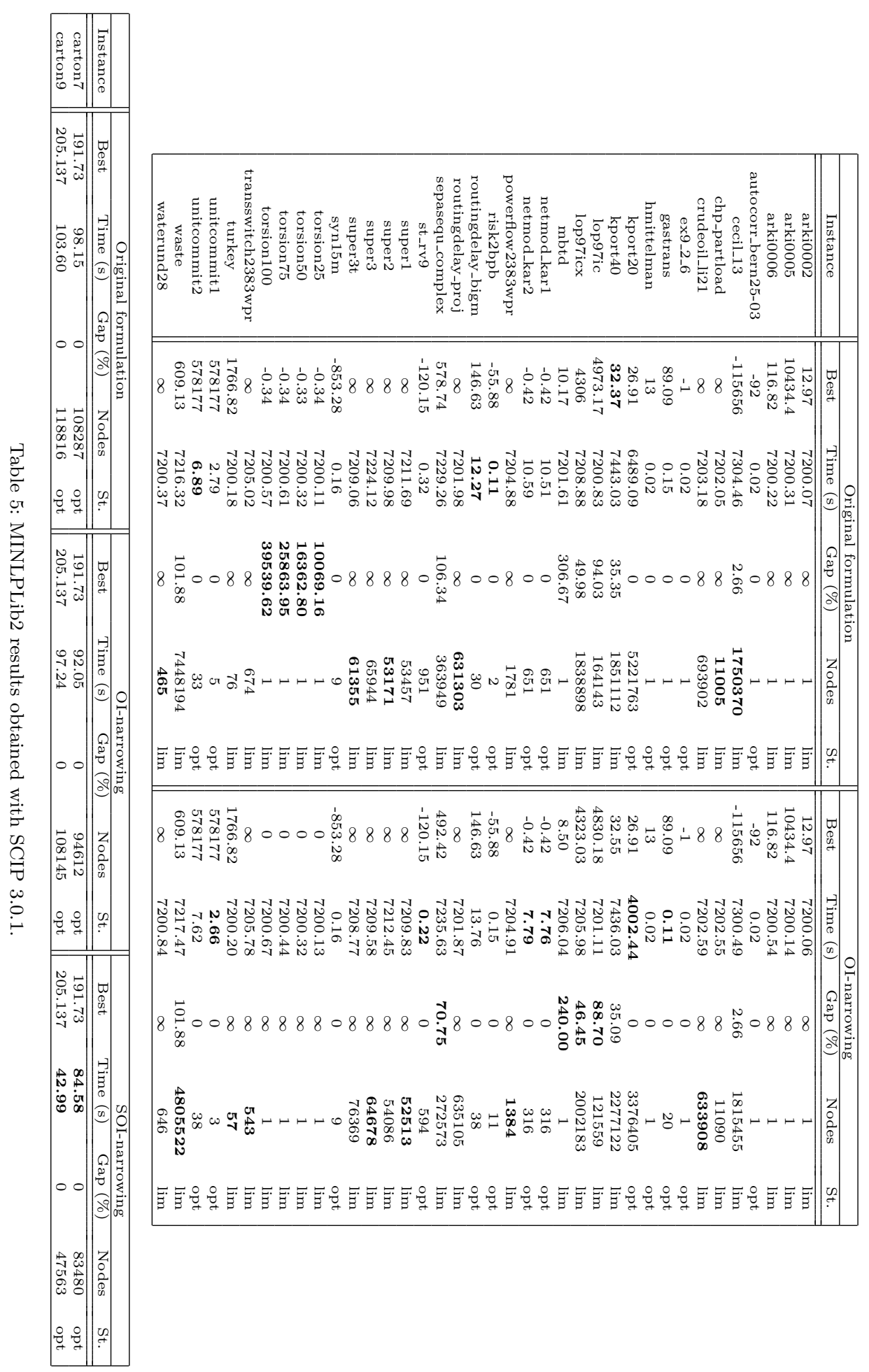




\begin{tabular}{|c||cc|cc|}
\hline & \multicolumn{2}{|c|}{ Original formulation } & \multicolumn{2}{c|}{ OI-narrowing } \\
\cline { 2 - 5 } Dataset & \# Best & Time $(\mathrm{s})$ & \# Best & Time $(\mathrm{s})$ \\
\hline \hline BQP & 21 & 42054.31 & 51 & 16.66 \\
\hline
\end{tabular}

Table 7: Aggregated solution statistics for the BQP dataset.

the OI narrowings. Table 8 exhibits detailed results. All narrowings were solved to optimality. As a side note, these results somehow support out claim that SBCs may eventually prevent $\mathrm{BB}$ algorithms to take correct decisions since some narrowings (21 in total) performed worse even under favorable conditions.

\begin{tabular}{|c|c|c|c|c|c|c|c|c|c|c|}
\hline & \multicolumn{5}{|c|}{ Original formulation } & \multicolumn{5}{|c|}{ OI-narrowing } \\
\hline Instance & Best & Time (s) & Gap (\%) & Nodes & St. & Best & Time (s) & Gap (\%) & Nodes & St. \\
\hline bqp_70_2xR & 580 & 7212.02 & 14.69 & 55721277 & $\lim$ & 580 & 0.14 & 0 & 0 & opt \\
\hline bqp_70_3xR & 1132 & 2.04 & 0 & 4644 & opt & 1132 & 0.58 & 0 & 613 & opt \\
\hline bqp_70_4x14 & 427 & 2.85 & 0 & 21397 & opt & 427 & 0.13 & 0 & 79 & opt \\
\hline bqp_70_4xR & 648 & 16.13 & 0 & 121652 & opt & 648 & 0.12 & 0 & 120 & opt \\
\hline bqp_70_5xR & 197 & 0.12 & 0 & 245 & opt & 197 & 0.17 & 0 & 47 & opt \\
\hline bqp_70_6x 10 & 155 & 0.68 & 0 & 3065 & opt & 155 & 0.13 & 0 & 116 & opt \\
\hline bqp_70_7xR & 112 & 0.05 & 0 & 163 & opt & 112 & 0.09 & 0 & 55 & opt \\
\hline bqp_70_9x7 & 70 & 0.02 & 0 & 49 & opt & 70 & 0.05 & 0 & 29 & opt \\
\hline bqp_75_2x25 & 763 & 0.07 & 0 & 141 & opt & 763 & 0.08 & 0 & 35 & opt \\
\hline bqp_75_2xR & 646 & 0.05 & 0 & 55 & opt & 646 & 0.11 & 0 & 0 & opt \\
\hline bqp_75_3xR & 651 & 0.06 & 0 & 111 & opt & 651 & 0.17 & 0 & 66 & opt \\
\hline bqp_75_4x15 & 949 & 14.61 & 0 & 126111 & opt & 949 & 0.20 & 0 & 71 & opt \\
\hline bqp_75_4xR & 981 & 0.26 & 0 & 442 & opt & 981 & 0.11 & 0 & 42 & opt \\
\hline bqp_-75_5x15 & 931 & 93.00 & 0 & 720167 & opt & 931 & 0.15 & 0 & 186 & opt \\
\hline bqp_75_5xR & 604 & 0.84 & 0 & 3901 & opt & 604 & 0.20 & 0 & 36 & opt \\
\hline bqp_75_6xR & 210 & 0.07 & 0 & 132 & opt & 210 & 0.12 & 0 & 58 & opt \\
\hline bqp_75_7xR & 172 & 0.06 & 0 & 176 & opt & 172 & 0.09 & 0 & 54 & opt \\
\hline bqp_75_8xR & 100 & 0.06 & 0 & 174 & opt & 100 & 0.08 & 0 & 42 & opt \\
\hline bqp_80_2x20 & 500 & 0.01 & 0 & 0 & opt & 500 & 0.02 & 0 & 0 & opt \\
\hline bqp_80_2xR & 1760 & 59.90 & 0 & 350488 & opt & 1760 & 0.06 & 0 & 0 & opt \\
\hline bqp_80_3x20 & 100 & 0.01 & 0 & 0 & opt & 100 & 0.02 & 0 & 0 & opt \\
\hline bqp_80_3xR & 853 & 0.25 & 0 & 371 & opt & 853 & 0.24 & 0 & 137 & opt \\
\hline bqp_80_4x16 & 976 & 75.01 & 0 & 549538 & opt & 976 & 0.18 & 0 & 61 & opt \\
\hline bqp_80_4xR & 715 & 3.42 & 0 & 20751 & opt & 715 & 0.19 & 0 & 29 & opt \\
\hline bqp_80_5x16 & 936 & 27.07 & 0 & 206700 & opt & 936 & 0.40 & 0 & 143 & opt \\
\hline bqp_80_5xR & 305 & 0.66 & 0 & 1696 & opt & 305 & 0.22 & 0 & 80 & opt \\
\hline bqp_80_6xR & 78 & 0.04 & 0 & 80 & opt & 78 & 0.12 & 0 & 30 & opt \\
\hline bqp_80_7x10 & 170 & 0.04 & 0 & 27 & opt & 170 & 0.07 & 0 & 12 & opt \\
\hline bqp_80_8xR & 100 & 0.06 & 0 & 59 & opt & 100 & 0.09 & 0 & 22 & opt \\
\hline bqp_85_12x5 & 147 & 1.25 & 0 & 5825 & opt & 147 & 0.66 & 0 & 1561 & opt \\
\hline bqp_85_16x5 & 69 & 1.76 & 0 & 10181 & opt & 69 & 1.06 & 0 & 3740 & opt \\
\hline bqp_85_2x17 & 2924 & 0.04 & 0 & 24 & opt & 2924 & 0.04 & 0 & 24 & opt \\
\hline bqp_85_2xR & 5189 & 3.98 & 0 & 19265 & opt & 5189 & 0.06 & 0 & 8 & opt \\
\hline bqp_85_3xR & 695 & 649.55 & 0 & 4322081 & opt & 695 & 0.10 & 0 & 22 & opt \\
\hline bqp_85_4x17 & 714 & 27.94 & 0 & 156926 & opt & 714 & 0.15 & 0 & 44 & opt \\
\hline bqp_85_4xR & 1374 & 61.84 & 0 & 477419 & opt & 1374 & 0.21 & 0 & 60 & opt \\
\hline bqp_85_5xR & 827 & 1.75 & 0 & 1942 & opt & 827 & 0.86 & 0 & 260 & opt \\
\hline bqp_85_6xR & 233 & 1.65 & 0 & 2463 & opt & 233 & 0.17 & 0 & 202 & opt \\
\hline bqp_85_7xR & 141 & 0.07 & 0 & 172 & opt & 141 & 0.06 & 0 & 64 & opt \\
\hline bqp_85_8xR & 160 & 0.65 & 0 & 346 & opt & 160 & 0.06 & 0 & 37 & opt \\
\hline bqp_85_9xR & 52 & 0.14 & 0 & 339 & opt & 52 & 0.10 & 0 & 110 & opt \\
\hline bqp_90_2x30 & 9420 & 7212.55 & 35.51 & 35350170 & $\lim$ & 9420 & 0.11 & 0 & 23 & opt \\
\hline bqp_90_2xR & 1872 & 7212.53 & 32.07 & 36978133 & $\lim$ & 1872 & 0.08 & 0 & 3 & opt \\
\hline bqp_90_3x30 & 6585 & 7212.67 & 34.19 & 33351114 & $\lim$ & 6585 & 0.16 & 0 & 58 & opt \\
\hline bqp_90_3xR & 2735 & 4.49 & 0 & 19974 & opt & 2735 & 0.18 & 0 & 12 & opt \\
\hline bqp_90_4x18 & 576 & 40.24 & 0 & 191424 & opt & 576 & 0.11 & 0 & 23 & opt \\
\hline bqp_90_4xR & 1047 & 3.22 & 0 & 14661 & opt & 1047 & 0.22 & 0 & 70 & opt \\
\hline bqp_90_5x15 & 225 & 0.01 & 0 & 0 & opt & 225 & 0.03 & 0 & 0 & opt \\
\hline bqp_90_5xR & 215 & 0.61 & 0 & 372 & opt & 215 & 0.26 & 0 & 89 & opt \\
\hline bqp_90_6xR & 183 & 0.12 & 0 & 168 & opt & 183 & 0.17 & 0 & 49 & opt \\
\hline bqp_90_7xR & 283 & 2.90 & 0 & 15893 & opt & 283 & 0.55 & 0 & 283 & opt \\
\hline bqp_90_8x10 & 925 & 65.20 & 0 & 411100 & opt & 925 & 1.17 & 0 & 2345 & opt \\
\hline bqp_90_9x9 & 117 & 0.04 & 0 & 53 & opt & 117 & 0.06 & 0 & 44 & opt \\
\hline bqp_95_18x5 & 95 & 2.22 & 0 & 16397 & opt & 95 & 1.40 & 0 & 4329 & opt \\
\hline bqp_95_2xR & 4226 & 3.26 & 0 & 14109 & opt & 4226 & 0.11 & 0 & 10 & opt \\
\hline bqp_95_3xR & 1843 & 8.24 & 0 & 43460 & opt & 1843 & 0.12 & 0 & 38 & opt \\
\hline bqp_95_4x19 & 636 & 29.29 & 0 & 188938 & opt & 636 & 0.09 & 0 & 69 & opt \\
\hline
\end{tabular}




\begin{tabular}{|c|c|c|c|c|c|c|c|c|c|c|}
\hline bqp_95_4xR & 480 & 0.08 & 0 & 9 & opt & 480 & 0.07 & 0 & 12 & opt \\
\hline bqp_95_5xR & 468 & 0.07 & 0 & 65 & opt & 468 & 0.37 & 0 & 50 & opt \\
\hline bqp_95_6xR & 220 & 0.05 & 0 & 1 & opt & 220 & 0.04 & 0 & 0 & opt \\
\hline bqp_95_7xR & 468 & 1.33 & 0 & 232 & opt & 468 & 0.17 & 0 & 27 & opt \\
\hline bqp_95_8xR & 1425 & 3194.67 & 0 & 23845041 & opt & 1425 & 0.12 & 0 & 0 & opt \\
\hline bqp_95_9xR & 209 & 1.06 & 0 & 2046 & opt & 209 & 0.29 & 0 & 243 & opt \\
\hline bqp_100_2xR & 8606 & 7211.99 & 43.86 & 28145015 & $\lim$ & 8606 & 0.32 & 0 & 8 & opt \\
\hline bqp_100_3x25 & 700 & 0.12 & 0 & 71 & opt & 700 & 0.07 & 0 & 0 & opt \\
\hline bqp_100_3xR & 6942 & 186.10 & 0 & 1036046 & opt & 6942 & 0.49 & 0 & 67 & opt \\
\hline bqp_100_4x20 & 4230 & 1238.27 & 0 & 7701248 & opt & 4230 & 0.22 & 0 & 99 & opt \\
\hline bqp_100_4xR & 400 & 1.36 & 0 & 5260 & opt & 400 & 0.16 & 0 & 61 & opt \\
\hline bqp_100_5x20 & 1280 & 161.01 & 0 & 971381 & opt & 1280 & 0.79 & 0 & 167 & opt \\
\hline bqp_100_5xR & 884 & 0.16 & 0 & 246 & opt & 884 & 0.31 & 0 & 33 & opt \\
\hline bqp_100_6xR & 725 & 0.09 & 0 & 61 & opt & 725 & 0.23 & 0 & 5 & opt \\
\hline bqp_100_7xR & 358 & 0.10 & 0 & 105 & opt & 358 & 0.17 & 0 & 25 & opt \\
\hline bqp_100_8xR & 294 & 0.16 & 0 & 184 & opt & 294 & 0.14 & 0 & 71 & opt \\
\hline bqp_100_9x10 & 70 & 0.02 & 0 & 0 & opt & 70 & 0.02 & 0 & 0 & opt \\
\hline
\end{tabular}

Table 8: BQP results obtained with CPLEX 12.6

Lastly, we ran a second round of tests restricted to the instances whose original formulations were not solved to optimality, now forcing CPLEX to unleash its full power in terms of symmetry breaking (parameter symmetry of CPLEX's API set to level 5). The results are presented in Table 9. Remarkably, there is no significant change in the final gaps, meaning that these instances are indeed hard to solve; except if one employs, for instance, the OI reformulations.

\begin{tabular}{|c||cccc|}
\hline \multicolumn{1}{|c||}{} & \multicolumn{4}{c|}{ Original formulation } \\
\cline { 2 - 5 } Instance & Best & Time (s) & Gap (\%) & St. \\
\hline \hline bqp_70_2xR & 580 & 7212.03 & 14.62 & lim \\
bqp_90_2x30 & 9420 & 7212.37 & 35.51 & lim \\
bqp_90_2xR & 1872 & 7212.13 & 32.07 & lim \\
bqp_90_3x30 & 6585 & 7212.88 & 34.19 & lim \\
bqp_100_2xR & 8606 & 7212.29 & 43.86 & lim \\
\hline
\end{tabular}

Table 9: Extended results obtained with CPLEX 12.6 for hard BQP instances.

\section{Conclusions}

In this paper we discussed the notion of Orbital Independence by presenting theoretical conditions that allow us to break symmetries from different orbits of mathematical programs concurrently: we introduced the concept of independent sets of orbits. An algorithm that potentially identifies the largest independent set of orbits of a mathematical program and generates SBCs to all orbits of such set was also presented. We evaluated the impact of our algorithm by conducting experiments with symmetric instances taken from the libraries MIPLIB2010 and MINLPLib2. We observed that the computational results were coherent in theoretical terms but average-to-poor in practical terms. We conjecture why the results are not expressive, but we take them mainly as an evidence of reaching the limit of what we can do in terms of SSB: no significant impact (for the general case) despite exploiting as many orbits as possible. It seems like determining automatically (prior to exploring the BB tree) a set of SBCs capable of producing a strong computational impact timewise is as hard as solving the original problem itself. Yet we consider the exploitation of OI ideas 
dynamically (e.g. by means of branching rules) as a potential improvement direction since DSB strategies seem to be most efficient ones. Finally, we have introduced a family of highly symmetric Binary Quadratic Programs which proved to be relevant to the OI theory since they purposely embed the conditions under which the usage of Symmetry-Breaking Constraints is majoritarily advantageous.

\section{Acknowledgments}

The first author (GD) was supported by a CNPq Ph.D. thesis award. The second author (LL) gratefully acknowledges funding from the European Union's Horizon 2020 research and innovation programme under the Marie Sklodowska-Curie grant agreement n. 764759. We would like to thank two anonymous referees for all the comments and suggestions that helped us to improve our work.

\section{References}

1. Achterberg, T.: SCIP: Solving constraint integer programs. Mathematical Programming Computation 1(1), 1-41 (2009)

2. Berstel, J., Boasson, L.: Context-free languages. In: J. van Leeuwen (ed.) Handbook of Theoretical Computer Science, vol. B, pp. 59-102. MIT Press, Cambridge, MA, USA (1990)

3. Bomze, I., Budinich, M., Pardalos, P., Pelillo, M.: The maximum clique problem. In: D.Z. Du, P. Pardalos (eds.) Handbook of Combinatorial Optimization: Supp. Vol. A, pp. 1-74. Springer US, Boston, MA (1998)

4. Costa, A., Hansen, P., Liberti, L.: Formulation symmetries in circle packing. In: R. Mahjoub (ed.) ISCO 2010 Proceedings, ENDM, vol. 36, pp. 1303-1310. Elsevier, Amsterdam, Netherlands (2010)

5. Dias, G., Liberti, L.: Orbital independence in symmetric mathematical programs. In: Z. Lu et al (ed.) COCOA 2015 Proceedings, LNCS, vol. 9486, pp. 467-480. Springer (2015)

6. Faenza, Y., Kaibel, V.: Extended formulations for packing and partitioning orbitopes. Mathematics of Operations Research 34(3), 686-697 (2009)

7. Fischetti, M., Liberti, L.: Orbital shrinking. In: R. Mahjoub, V. Markakis, I. Milis, V. Paschos (eds.) ISCO 2012 Proceedings, LNCS, vol. 7422, pp. 48-58. Springer, Berlin, Heidelberg (2012)

8. Fischetti, M., Liberti, L., Salvagnin, D., Walsh, T.: Orbital shrinking: theory and applications. Discrete Applied Mathematics 222, 109-123 (2017)

9. Fourer, R., Gay, D., Kernighan, B.: The AMPL Book, second edition edn. Cengage Learning, California, USA (2002)

10. Friedman, E.: Fundamental domains for integer programs with symmetries. In: A. Dress, Y. Xu, B. Zhu (eds.) COCOA 2007 Proceedings, LNCS, vol. 4616, pp. 146-153. Springer (2007)

11. Galli, S.: Parsing AMPL internal format for linear and non-linear expressions (2004). B.Sc. dissertation, DEI, Politecnico di Milano, Italy

12. Gallo, G., Hammer, P., Simeone, B.: Quadratic knapsack problems, pp. 132-149. Springer Berlin Heidelberg, Berlin, Heidelberg (1980)

13. IBM: ILOG CPLEX 12.6 - User's manual (2014)

14. Kaibel, V., Pfetsch, M.: Packing and partitioning orbitopes. Mathematical Programming 114(1), 1-36 (2008)

15. Liberti, L.: Reformulations in mathematical programming: Definitions and systematics. RAIRO-RO 43(1), 55-86 (2009)

16. Liberti, L.: Reformulations in mathematical programming: Automatic symmetry detection and exploitation. Mathematical Programming A 131, 273-304 (2012)

17. Liberti, L.: Symmetry in mathematical programming. In: S. Leyffer, J. Lee (eds.) Mixed Integer Nonlinear Programming, IMA Series, vol. 154, pp. 263-286. Springer, New York (2012) 
18. Liberti, L., Cafieri, S., Savourey, D.: Reformulation optimization software engine. In: K. Fukuda et al (ed.) Mathematical Software, LNCS, vol. 6327, pp. 303-314. Springer (2010)

19. Liberti, L., Ostrowski, J.: Stabilizer-based symmetry breaking constraints for mathematical programs. Journal of Global Optimization 60, 183-194 (2014)

20. Margot, F.: Pruning by isomorphism in branch-and-cut. Mathematical Programming 94, 71-90 (2002)

21. Margot, F.: Exploiting orbits in symmetric ILP. Mathematical Programming B 98, 3-21 (2003)

22. Margot, F.: Symmetry in integer linear programming. In: M. Jünger et al (ed.) 50 Years of Integer Programming, pp. 647-681. Springer, Berlin (2010)

23. McKay, B.: Practical graph isomorphism. Congressus Numerantium 30, 45-87 (1981)

24. McKay, B., Piperno, A.: Practical graph isomorphism, II. Journal of Symbolic Computation 60, 94-112 (2014)

25. Ostrowski, J., Linderoth, J., Rossi, F., Smriglio, S.: Orbital branching. Mathematical Programming 126(1), 147-178 (2011)

26. Pfetsch, M., Rehn, T.: A computational comparison of symmetry handling methods for mixed integer programs. Tech. Rep. 5209, Optimization Online (2015)

27. The GAP Group: GAP - Groups, Algorithms and Programming. Version 4.7.4 (2014) 